\title{
Strong compact formalism for characteristic boundary conditions with discontinuous spectral methods
}

\author{
Romain Fiévet ${ }^{\mathrm{a}, *}$, Hughes Deniau ${ }^{\mathrm{a}}$, Estelle Piot $^{\mathrm{a}}$ \\ ${ }^{a}$ The French Aerospace Laboratory (ONERA/DMPE) - Université de Toulouse, F-31055, Toulouse, France
}

\begin{abstract}
Characteristic boundary conditions for the Navier-Stokes equations (NSCBC) are implemented for the first time with discontinuous spectral methods, namely the spectral difference and flux reconstruction. The implementation makes use of the resolution by these methods of the strong form of the Navier-Stokes equations by applying these conditions through a flux balance regularization which takes the form of a generalized element-compact correction polynomial. It is shown to be at least as effective as similar implementations in finite volume solvers, and sustains arbitrarily-high orders of accuracy on hexahedral-based unstructured meshes. Further, Navier-Stokes time-domain impedance boundary conditions are derived and implemented as a NSCBC sub-class. They account for the diffusive process at the wall and are shown to properly resolve broadband impedance models under normal and grazing flow conditions. The ability of these NSCBC in preventing the appearance of spurious reflections at the boundaries is demonstrated through a varied series of bench-marking simulations. They effectively shield the inner computational domain from any far-field unphysical contamination. Overall, this work enables the use of strong discontinuous spectral methods to study unsteady problems on complex geometries.
\end{abstract}

Keywords: Spectral difference; Flux reconstruction; Navier-Stokes characteristic boundary conditions; Time-domain impedance boundary conditions

\section{Introduction}

Discontinuous spectral methods have gained much traction in the past years thanks to their ability to achieve locally arbitrarily-high orders of accuracy on unstructured meshes. Such accuracy was previously only attainable with large-stencil finite difference (FD) schemes naturally restricted to structured grids, which prevented the use of complex geometries with high-order schemes. High-order schemes permit to accurately resolve physical phenomenon where a broad range of wavelengths is relevant to the underlying physics and impacts the final result. For instance, they have been successfully used in computational aeroacoustics studies $[1,2,3]$, or in resolving thermo-acoustic $[4,5,6]$ and boundary layer $[7,8,9,10]$ instabilities growth.

The most celebrated family of discontinuous methods is the discontinuous Galerkin (DG) method introduced by Reed and Hill [11]. The review from Cockburn and Shu [12] highlights the development of the method to resolve hyperbolic systems and particularly the Navier-Stokes equations. The method has now reached a certain level of maturity and been used to perfom large-eddy simulations of complex problems such as detonation waves [13, 14] and laminar-turbulent transition over 3D airfoils [7,9] or in free-shear flows [10]. Further, characteristic boundary conditions have also recently been used with the DG method by Toulopoulos and Ekaterinaris [15]. First introduced by Thompson [16] for hyperbolic systems and later extended to Navier-Stokes equations by Poinsot and Lele [17], these are referred to as NSCBC (Navier-Stokes Characteristic Boundary Conditions).

The importance of such boundary conditions to computational fluid dynamics is two-fold. First, over-specifying a boundary condition typically induces numerical instabilities while under-specifying it feeds insufficient information to the computational domain. A well-behave boundary condition prescribes the correct amount of information based

${ }^{*}$ Corresponding author: fievetromain@gmail.com 
on the local flow speed [17]. Second, simpler boundary conditions such as Dirichlet or Neumann conditions are wellknown to cause spurious reflections from the boundaries back into the computational domain. Specifically, acoustic waves will reflect on an isobaric outflow boundary. As these nonphysical reflections interact with the inner domain's solution, they can potentially affect other modes relevant to the problem studied. For instance, aeroacoustics and transitional studies require non-reflecting boundary conditions to prevent the acoustic field and unstable modes' growth being polluted by these waves. As such, the implementation of characteristic non-reflecting boundary condition is a prerequisite to perform highly-accurate simulations.

Another class of NSCBC of strong interest to this study is the time-domain impedance boundary condition (TDIBC) [18]. It consists in resolving the amplitude and phase of a reflected acoustic wave as a frequency-dependent function of an incident wave upon its interaction with a porous surface. The value of such complex numerical boundary conditions lies in the design and optimization of coatings absorbing target frequencies of interest. For instance, acoustically-reacting surfaces are used in noise-canceling devices such as acoustic liners [18, 19]. Another promising application of such coatings is the canceling of unstable thermo-acoustic modes in combustion chambers [20, 21] or boundary layers $[22,23,24]$. Interestingly, TDIBC can mimick any sort of arbitrarily-complex condition (pure-delay, fully/partially reflecting) provided its analogous impedance be determined and serve a universal purpose. In all the aforementioned applications, it is crucial to use high-order numerical schemes to limit dissipative and dispersive errors and correctly resolve the modes growth and interactions. Hence, high-order methods and NSCBC/TDIBC appear to be highly synergetic. This provides a strong incentive in developing TDIBC for the Navier-Stokes equations (NSE) with discontinuous spectral methods.

However, the computational cost of the DG method tends to drastically increase with its order. This is due to the method resolving the weak integral form of the NSE in a finite-volume fashion. Hence, complex and costly quadrature rules are required to maintain a high order of accuracy. To cope with this issue, new methods have recently emerged which instead resolve the strong form of the NSE. They are referred to as strong discontinuous spectral methods. They are currently comprised of two family of schemes: the spectral difference (SD) and flux reconstruction (FR) methods.

The SD method has been pioneered by Kopriva and Kolias [25]. The core concept consists in constructing a polynomial representation of the solution inside each element in order to evaluate the flux and its derivatives along each coordinate. The order of the polynom, $q$, determines the scheme's order of accuracy which equals $q+1$. This method has been successfully used with unstructured meshes with Euler [26, 27] and later Navier-Stokes equations $[28,29]$. The numerical stability of the method was verified by Van den Abeele et al. [30] and Jameson [31], showing that the linear stability did not depend on the placement scheme of the solution points inside the elements. Finally, it has successfully been used to perform LES and DNS alike [32, 33, 34]. Vanharen et al. [35] have assessed the accuracy of the SD method for various polynomial orders and compared it with spectral-like resolution $6^{\text {th }}$-order compact FD schemes $[36,37]$. They showed that the $6^{\text {th }}$-order SD method is at least as accurate as large-stencil FD methods while offering the compactness suited to unstructured grids. A spectral-like resolution implies that the numerical scheme causes minimal dissipation and dispersion errors. This makes the SD a suitable candidate to develop a high-order numerical flow solver aimed at resolving complex physical problems and geometries alike.

In an effort to alleviate the computational and memory costs of the SD staggered approach, another strong discontinuous spectral method is called the flux reconstruction (FR) scheme. It directly evaluates the fluxes at the solution points and does not require a staggered formulation, which alleviates the memory cost. It was introduced by Huynh [38] and has since then been undergoing development. It has been adapted to multi-dimensional Navier-Stokes $[39,40]$ and another class of reconstruction polynomial has been introduced [41]. Notably, it has been successfully used to perform multi-dimensional large scale simulations for aerodynamics [42] and aeroacoustics [43]. While it is the newest method, it shows great potential compared to the disconstinuous Galerkin and spectral difference [44, 45] schemes.

However, NSCBC have yet to be enacted with SD and FR in order to truly enable their widespread use in largescale numerical simulations in the aforementioned fields and even beyond. In fact, any computational study of unsteady physical processes requires a sterilized numerical environment be used, as shielded as possible from far-field contamination. Otherwise, the use of a minimally-dissipative scheme inside the computational domain might prove counter-productive if time-growing parasite waves pollute it and interact with the process being scrutinized. In a subsonic flow, these perturbations take the form of back-traveling acoustic waves, whichever the nature of the source (combustion, turbulence, etc). The scope of the paper is to address this gap in the literature and implement NSCBC with both the SD and FR methods for the multi-dimensional Navier-Stokes equations. The derivation of these condi- 
tions in a generalized compact polynomial form compatible with these strong discontinuous methods constitutes the main novelty of this study. The SD method is the primary focus of the study and will be used to perform a set of bench-marking simulations assessing the effectiveness of the NSCBC implementation.

The paper is organized as follows. In Sec. 2, the characteristic Navier-Stokes equations are derived in generalized strong form (i.e. suitable for SD and FR). They are then further reduced into a more compact formulation which alleviates the computational cost for these methods. In Sec. 3, various types of NSCBC, referred to as sub-classes, are presented. Namely, the time-domain impedance condition is thoroughly presented. In Sec. 4, the implementation of these NSCBC in polynomial form with the SD and FR methods is detailed. In Sec. 5, typical bench-marking cases are run using a SD code to verify the said implementation. Last, the results of this study are summarized and conclusions are drawn.

For the sake of clarity, all arrays and vectors are highlighted in bold throughout the manuscript.

\section{Strong compact formalism for characteristic boundary conditions}

This section presents the derivation of the classical NSCBC pioneered by Poinsot and Lele [17] for generalized coordinates (i.e. pertaining to the class of unstructured meshes). It presents their rewriting into compact form, as will be used in Sec. 3 to be implemented within the framework of strong discontinuous spectral methods.

\subsection{Generalized characteristic form of the Navier Stokes equations}

As mentioned in Sec. 1, the strength of discontinuous spectral methods lies in their ability to retain an arbitrarilyhigh numerical order of accuracy on generic unstructured meshes. Specifically, the SD and FR methods which are investigated in this study resolve the strong conservative form of the NSE in three-dimensional (3D) generalized isoparametric coordinates $(\xi, \eta, \zeta)$. The transformation from the physical $\mathbf{x}=(x, y, z)$ space into the $\boldsymbol{\xi}=(\xi, \eta, \zeta)$ space is determined by the Jacobian of the transformation which equals $\xi_{\mathbf{x}}$ as given in the appendix. The conservation equations read in divergent form, for a viscous compressible non-reacting flow, as:

$$
\begin{aligned}
\frac{1}{J} \frac{\partial \mathbf{U}}{\partial t} & +\frac{\partial}{\partial \xi}\left(\mathbf{E}_{c} \frac{\xi_{x}}{J}+\mathbf{F}_{c} \frac{\xi_{y}}{J}+\mathbf{G}_{c} \frac{\xi_{z}}{J}\right)+\frac{\partial}{\partial \xi}\left(\mathbf{E}_{d} \frac{\xi_{x}}{J}+\mathbf{F}_{d} \frac{\xi_{y}}{J}+\mathbf{G}_{d} \frac{\xi_{z}}{J}\right) \\
& +\frac{\partial}{\partial \eta}\left(\mathbf{E}_{c} \frac{\eta_{x}}{J}+\mathbf{F}_{c} \frac{\eta_{y}}{J}+\mathbf{G}_{c} \frac{\eta_{z}}{J}\right)+\frac{\partial}{\partial \eta}\left(\mathbf{E}_{d} \frac{\eta_{x}}{J}+\mathbf{F}_{d} \frac{\eta_{y}}{J}+\mathbf{G}_{d} \frac{\eta_{z}}{J}\right) \\
& +\frac{\partial}{\partial \zeta}\left(\mathbf{E}_{c} \frac{\zeta_{x}}{J}+\mathbf{F}_{c} \frac{\zeta_{y}}{J}+\mathbf{G}_{c} \frac{\zeta_{z}}{J}\right)+\frac{\partial}{\partial \zeta}\left(\mathbf{E}_{d} \frac{\zeta_{x}}{J}+\mathbf{F}_{d} \frac{\zeta_{y}}{J}+\mathbf{G}_{d} \frac{\zeta_{z}}{J}\right)=0
\end{aligned}
$$

where $\mathbf{U}$ is the vector of conservative variables $\left(\rho, \rho u, \rho v, \rho w, \rho\left(e+|\mathbf{u}|^{2} / 2\right)^{\top}\right.$ with density $\rho\left[\mathrm{kg} \cdot \mathrm{m}^{-3}\right]$ and velocity $\mathbf{u}=(u, v, w)^{\top}\left[\mathrm{m} \cdot \mathrm{s}^{-1}\right]$ along physical coordinates $(x, y, z) . \rho e$ is the gas internal energy evaluated as a function of its temperature $T[\mathrm{~K}]$ and pressure $p[\mathrm{~Pa}]$ assuming a calorically perfect gas. $J$ is the determinant of the transformation matrix from physical to iso-parametric coordinates given in the appendix. The convective and diffusive fluxes of $\mathbf{U}$ in physical space are respectively called $\left(\mathbf{E}_{c}, \mathbf{F}_{c}, \mathbf{G}_{c}\right)$ and $\left(\mathbf{E}_{d}, \mathbf{F}_{d}, \mathbf{G}_{d}\right)$. They read as:

$$
\begin{aligned}
& \mathbf{E}_{c}=\left(\rho u, \rho u^{2}+p, \rho v u, \rho w u,(\rho e+p) u\right)^{\top} \\
& \mathbf{F}_{c}=\left(\rho v, \rho u v, \rho v^{2}+p, \rho w v,(\rho e+p) v\right)^{\top} \\
& \mathbf{G}_{c}=\left(\rho w, \rho u w, \rho v w, \rho w^{2}+p,(\rho e+p) w\right)^{\top} \\
& \mathbf{E}_{d}=\left(0, p+\tau_{(1,1)}, \tau_{(2,1)}, \tau_{(3,1)}, u \tau_{(1,1)}+v \tau_{(2,1)}+w \tau_{(3,1)}+\lambda_{T} \partial_{x} T\right)^{\top} \\
& \mathbf{F}_{d}=\left(0, \tau_{(1,2)}, p+\tau_{(2,2)}, \tau_{(3,2)}, u \tau_{(1,2)}+v \tau_{(2,2)}+w \tau_{(3,2)}+\lambda_{T} \partial_{y} T\right)^{\top} \\
& \mathbf{G}_{d}=\left(0, \tau_{(1,3)}, \tau_{(2,3)}, p+\tau_{(3,3)}, u \tau_{(1,3)}+v \tau_{(2,3)}+w \tau_{(3,3)}+\lambda_{T} \partial_{z} T\right)^{\top}
\end{aligned}
$$

with viscous stress tensor $\tau_{(i, j)}\left[\mathrm{kg} \cdot \mathrm{m}^{-1} \cdot \mathrm{s}^{-2}\right]$ and thermal conductivity $\lambda_{T}\left[\mathrm{~J} \cdot \mathrm{K}^{-1}\right]$. The derivation of the system of equations governing the NSCBC in generalized coordinates follows that of Kim and Joo [46]. It consists in modifying 
the flux balance at the boundaries to account for the NSCBC. While originally intended for FD schemes, their method is shown to be fully compatible with strong discontinuous spectral methods as well. Conversely, the simple chainderivative rule permits to recast the three-dimensional (3D) non-reacting NSE, written in physical coordinates as:

$$
\frac{\partial \mathbf{U}}{\partial t}+\frac{\partial \mathbf{E}_{c}}{\partial x}+\frac{\partial \mathbf{F}_{c}}{\partial y}+\frac{\partial \mathbf{G}_{c}}{\partial z}+\frac{\partial \mathbf{E}_{d}}{\partial x}+\frac{\partial \mathbf{F}_{d}}{\partial y}+\frac{\partial \mathbf{G}_{d}}{\partial z}=0
$$

into the following form:

$$
\begin{aligned}
\frac{\partial \mathbf{U}}{\partial t} & +\left(\xi_{x} \frac{\partial \mathbf{E}_{c}}{\partial \xi}+\xi_{y} \frac{\partial \mathbf{F}_{c}}{\partial \xi}+\xi_{z} \frac{\partial \mathbf{G}_{c}}{\partial \xi}\right)+\left(\xi_{x} \frac{\partial \mathbf{E}_{d}}{\partial \xi}+\xi_{y} \frac{\partial \mathbf{F}_{d}}{\partial \xi}+\xi_{z} \frac{\partial \mathbf{G}_{d}}{\partial \xi}\right) \\
& +\left(\eta_{x} \frac{\partial \mathbf{E}_{c}}{\partial \eta}+\eta_{y} \frac{\partial \mathbf{F}_{c}}{\partial \eta}+\eta_{z} \frac{\partial \mathbf{G}_{c}}{\partial \eta}\right)+\left(\eta_{x} \frac{\partial \mathbf{E}_{d}}{\partial \eta}+\eta_{y} \frac{\partial \mathbf{F}_{d}}{\partial \eta}+\eta_{z} \frac{\partial \mathbf{G}_{d}}{\partial \eta}\right) \\
& +\left(\zeta_{x} \frac{\partial \mathbf{E}_{c}}{\partial \zeta}+\zeta_{y} \frac{\partial \mathbf{F}_{c}}{\partial \zeta}+\zeta_{z} \frac{\partial \mathbf{G}_{c}}{\partial \zeta}\right)+\left(\zeta_{x} \frac{\partial \mathbf{E}_{d}}{\partial \zeta}+\zeta_{y} \frac{\partial \mathbf{F}_{d}}{\partial \zeta}+\zeta_{z} \frac{\partial \mathbf{G}_{d}}{\partial \zeta}\right)=0
\end{aligned}
$$

It is important to note that Eqs. (4) and (1) are equivalent due to the mesh transformation's invariants verifying:

$$
\begin{aligned}
0 & =\left[\frac{\partial}{\partial \xi}\left(\frac{\xi_{x}}{J}\right)+\frac{\partial}{\partial \eta}\left(\frac{\eta_{x}}{J}\right)+\frac{\partial}{\partial \zeta}\left(\frac{\zeta_{x}}{J}\right)\right] \\
& =\left[\frac{\partial}{\partial \xi}\left(\frac{\xi_{y}}{J}\right)+\frac{\partial}{\partial \eta}\left(\frac{\eta_{y}}{J}\right)+\frac{\partial}{\partial \zeta}\left(\frac{\zeta_{y}}{J}\right)\right] \\
& =\left[\frac{\partial}{\partial \xi}\left(\frac{\xi_{z}}{J}\right)+\frac{\partial}{\partial \eta}\left(\frac{\eta_{z}}{J}\right)+\frac{\partial}{\partial \zeta}\left(\frac{\zeta_{z}}{J}\right)\right]
\end{aligned}
$$

Without any loss of generality, let us consider a boundary located at a $\xi$-normal face, i.e. at the end of the computational domain along the generalized coordinate $\xi$. The characteristic decomposition of the $\xi$-convective flux is expressed as:

$$
\left(\xi_{x} \frac{\partial \mathbf{E}_{c}}{\partial \xi}+\xi_{y} \frac{\partial \mathbf{F}_{c}}{\partial \xi}+\xi_{z} \frac{\partial \mathbf{G}_{c}}{\partial \xi}\right)=P_{\mathbf{U}} \Delta P_{\mathbf{U}}^{-1} \frac{\partial \mathbf{U}}{\partial \xi}
$$

with $\Delta$ being the diagonalized matrix of $\partial_{\mathbf{U}} \mathbf{E}_{c}$ 's eigenvalues. In $3 \mathrm{D}, \Delta=\left(u_{n}, u_{n}, u_{n}, u_{n}+c, u_{n}-c\right)$ with $c$ being the local speed of sound and $u_{n}$ the face normal velocity calculated as $u_{n}=\mathbf{u} . \mathbf{n}=u \cdot n_{x}+v \cdot n_{y}+w \cdot n_{z}$. This corresponds to three entropy waves (which includes vorticity), one forward-traveling acoustic wave and one backward-traveling acoustic wave. The face's normal vector $\mathbf{n}$ is evaluated from the metrics as $\nabla \boldsymbol{\xi} /|\nabla \boldsymbol{\xi}| . P_{\mathbf{U}}$ is the transformation matrix from conservative to characteristic space and is given in the appendix for the sake of completeness. The vector $\mathcal{L}$ corresponds to the strength of the $\xi$-convective characteristics and is defined as:

$$
\mathcal{L}=\Delta P_{\mathbf{U}}^{-1} \frac{\partial \mathbf{U}}{\partial \xi}=P_{\mathbf{U}}^{-1}\left(\xi_{x} \frac{\partial \mathbf{E}_{c}}{\partial \xi}+\xi_{y} \frac{\partial \mathbf{F}_{c}}{\partial \xi}+\xi_{z} \frac{\partial \mathbf{G}_{c}}{\partial \xi}\right) .
$$

Likewise, the transformation matrix is used to define the following quantities:

$$
\begin{aligned}
\partial \mathbf{W} & =P_{\mathbf{U}}^{-1} \partial \mathbf{U} \\
\mathcal{T}_{c} & =P_{\mathbf{U}}^{-1}\left[\left(\eta_{x} \frac{\partial \mathbf{E}_{c}}{\partial \eta}+\eta_{y} \frac{\partial \mathbf{F}_{c}}{\partial \eta}+\eta_{z} \frac{\partial \mathbf{G}_{c}}{\partial \eta}\right)+\left(\zeta_{x} \frac{\partial \mathbf{E}_{c}}{\partial \zeta}+\zeta_{y} \frac{\partial \mathbf{F}_{c}}{\partial \zeta}+\zeta_{z} \frac{\partial \mathbf{G}_{c}}{\partial \zeta}\right)\right] \\
\mathcal{T}_{d} & =P_{\mathbf{U}}^{-1}\left[\left(\eta_{x} \frac{\partial \mathbf{E}_{d}}{\partial \eta}+\eta_{y} \frac{\partial \mathbf{F}_{d}}{\partial \eta}+\eta_{z} \frac{\partial \mathbf{G}_{d}}{\partial \eta}\right)+\left(\zeta_{x} \frac{\partial \mathbf{E}_{d}}{\partial \zeta}+\zeta_{y} \frac{\partial \mathbf{F}_{d}}{\partial \zeta}+\zeta_{z} \frac{\partial \mathbf{G}_{d}}{\partial \zeta}\right)\right], \\
\mathcal{D} & =P_{\mathbf{U}}^{-1}\left(\xi_{x} \frac{\partial \mathbf{E}_{d}}{\partial \xi}+\xi_{y} \frac{\partial \mathbf{F}_{d}}{\partial \xi}+\xi_{z} \frac{\partial \mathbf{G}_{d}}{\partial \xi}\right) .
\end{aligned}
$$


Hence, Eq. (4) can be rewritten in characteristic space as:

$$
\frac{\partial \mathbf{W}}{\partial t}+\mathcal{L}+\mathcal{T}_{c}+\mathcal{T}_{d}+\mathcal{D}=0
$$

Importantly, $\partial \mathbf{W}_{1,2,3}$ correspond to the entropy waves propagating at velocity u.n while $\partial W_{+}$(respectively $\partial W_{-}$) is the $\mathbf{u} . \mathbf{n}+c$ (respectively $\mathbf{u} . \mathbf{n}-c)$ propagating acoustic wave. They read as:

$$
\left\{\begin{array}{l}
\partial W_{1}=n_{x} \partial \rho+n_{z} \partial v-n_{y} \partial w-\left(n_{x} / c^{2}\right) \partial p \\
\partial W_{2}=n_{y} \partial \rho-n_{z} \partial u+n_{x} \partial w-\left(n_{y} / c^{2}\right) \partial p \\
\partial W_{3}=n_{z} \partial \rho+n_{y} \partial u-n_{x} \partial v-\left(n_{z} / c^{2}\right) \partial p \\
\partial W_{+}=\left(n_{x} \partial u+n_{y} \partial v+n_{z} \partial w\right) \rho c+\partial p \\
\partial W_{-}=\left(-n_{x} \partial u-n_{y} \partial v-n_{z} \partial w\right) \rho c+\partial p
\end{array}\right.
$$

The contribution from the convective and diffusive tangent fluxes, $\mathcal{T}_{c}$ and $\mathcal{T}_{d}$, are evaluated from the inner computational domain's solution. The diffusive flux $\mathcal{D}$ usually relies on Dirichlet or Neumann conditions to calculate the momentum and thermal energy gradients.

Let us now introduce $\widehat{\mathbf{E}}_{c}, \widehat{\mathbf{F}}_{c}$ and $\widehat{\mathbf{G}}_{c}$ alongside $\widehat{\mathbf{E}}_{d}, \widehat{\mathbf{F}}_{d}$ and $\widehat{\mathbf{G}}_{d}$ which read as:

$$
\left\{\begin{array}{l}
\widehat{\mathbf{E}}_{c}=\mathbf{E}_{c} \frac{\xi_{x}}{J}+\mathbf{F}_{c} \frac{\xi_{y}}{J}+\mathbf{G}_{c} \frac{\xi_{z}}{J} \\
\widehat{\mathbf{F}}_{c}=\mathbf{E}_{c} \frac{\eta_{x}}{J}+\mathbf{F}_{c} \frac{\eta_{y}}{J}+\mathbf{G}_{c} \frac{\eta_{z}}{J} \\
\widehat{\mathbf{G}}_{c}=\mathbf{E}_{c} \frac{\zeta_{x}}{J}+\mathbf{F}_{c} \frac{\xi_{y}}{J}+\mathbf{G}_{c} \frac{\zeta_{z}}{J} \\
\widehat{\mathbf{E}}_{d}=\mathbf{E}_{d} \frac{\xi_{x}}{J}+\mathbf{F}_{d} \frac{\xi_{y}}{J}+\mathbf{G}_{d} \frac{\xi_{z}}{J} \\
\widehat{\mathbf{F}}_{d}=\mathbf{E}_{d} \frac{\eta_{x}}{J}+\mathbf{F}_{d} \frac{\eta_{y}}{J}+\mathbf{G}_{d} \frac{\eta_{z}}{J} \\
\widehat{\mathbf{G}}_{d}=\mathbf{E}_{d} \frac{\zeta_{x}}{J}+\mathbf{F}_{d} \frac{\zeta_{y}}{J}+\mathbf{G}_{d} \frac{\zeta_{z}}{J}
\end{array}\right.
$$

Equation (1) is further reduced to:

$$
\frac{1}{J} \frac{\partial \mathbf{U}}{\partial t}+\frac{\partial \widehat{\mathbf{E}}_{c}}{\partial \xi}+\frac{\partial \widehat{\mathbf{F}}_{c}}{\partial \eta}+\frac{\partial \widehat{\mathbf{G}}_{c}}{\partial \zeta}+\frac{\partial \widehat{\mathbf{E}}_{d}}{\partial \xi}+\frac{\partial \widehat{\mathbf{F}}_{d}}{\partial \eta}+\frac{\partial \widehat{\mathbf{G}}_{d}}{\partial \zeta}=0,
$$

The equivalence between Eq. (4) and Eq. (12) yields the following expression for $\mathcal{L}$ :

$$
\begin{aligned}
\mathcal{L} & =P_{\mathbf{U}}^{-1} J\left(\frac{\xi_{x}}{J} \frac{\partial \mathbf{E}_{c}}{\partial \xi}+\frac{\xi_{y}}{J} \frac{\partial \mathbf{F}_{c}}{\partial \xi}+\frac{\xi_{z}}{J} \frac{\partial \mathbf{E}_{c}}{\partial \xi}\right) \\
& =P_{\mathbf{U}}^{-1} J\left(\frac{\partial \widehat{\mathbf{E}}_{c}}{\partial \xi}-\left[\mathbf{E}_{c} \frac{\partial}{\partial \xi}\left(\frac{\xi_{x}}{J}\right)+\mathbf{F}_{c} \frac{\partial}{\partial \xi}\left(\frac{\xi_{y}}{J}\right)+\mathbf{G}_{c} \frac{\partial}{\partial \xi}\left(\frac{\xi_{z}}{J}\right)\right]\right) \\
& =P_{\mathbf{U}}^{-1} J\left(\frac{\partial \widehat{\mathbf{E}}_{c}}{\partial \xi}-\mathcal{A}_{c}(\xi)\right)
\end{aligned}
$$

The additional term shown between brackets, $\mathcal{A}_{c}(\xi)$, accounts for the mesh's non-orthogonality. Similarly, the following quantities are defined:

$$
\begin{aligned}
\mathcal{D} & =P_{\mathbf{U}}^{-1} J\left(\frac{\partial \widehat{\mathbf{E}}_{d}}{\partial \xi}-\left[\mathbf{E}_{d} \frac{\partial}{\partial \xi}\left(\frac{\xi_{x}}{J}\right)+\mathbf{F}_{d} \frac{\partial}{\partial \xi}\left(\frac{\xi_{y}}{J}\right)+\mathbf{G}_{d} \frac{\partial}{\partial \xi}\left(\frac{\xi_{z}}{J}\right)\right]\right) \\
& =P_{\mathbf{U}}^{-1} J\left(\frac{\partial \widehat{\mathbf{E}}_{d}}{\partial \xi}-\mathcal{A}_{d}(\xi)\right)
\end{aligned}
$$




$$
\begin{aligned}
\mathcal{T}_{c} & =P_{\mathbf{U}}^{-1} J\left(\frac{\partial \widehat{\mathbf{F}}_{c}}{\partial \eta}-\left[\mathbf{E}_{c} \frac{\partial}{\partial \eta}\left(\frac{\eta_{x}}{J}\right)+\mathbf{F}_{c} \frac{\partial}{\partial \eta}\left(\frac{\eta_{y}}{J}\right)+\mathbf{G}_{c} \frac{\partial}{\partial \eta}\left(\frac{\eta_{z}}{J}\right)\right]\right) \\
& +P_{\mathbf{U}}^{-1} J\left(\frac{\partial \widehat{\mathbf{G}}_{c}}{\partial \zeta}-\left[\mathbf{E}_{c} \frac{\partial}{\partial \zeta}\left(\frac{\zeta_{x}}{J}\right)+\mathbf{F}_{c} \frac{\partial}{\partial \zeta}\left(\frac{\zeta_{y}}{J}\right)+\mathbf{G}_{c} \frac{\partial}{\partial \zeta}\left(\frac{\zeta_{z}}{J}\right)\right]\right) \\
& =P_{\mathbf{U}}^{-1} J\left(\frac{\partial \widehat{\mathbf{F}}_{c}}{\partial \eta}+\frac{\partial \widehat{\mathbf{G}}_{c}}{\partial \zeta}-\left[\mathcal{A}_{c}(\eta)+\mathcal{A}_{c}(\zeta)\right]\right),
\end{aligned}
$$

and

$$
\begin{aligned}
\mathcal{T}_{d} & =P_{\mathbf{U}}{ }^{-1} J\left(\frac{\partial \widehat{\mathbf{F}}_{d}}{\partial \eta}-\left[\mathbf{E}_{d} \frac{\partial}{\partial \eta}\left(\frac{\eta_{x}}{J}\right)+\mathbf{F}_{d} \frac{\partial}{\partial \eta}\left(\frac{\eta_{y}}{J}\right)+\mathbf{G}_{d} \frac{\partial}{\partial \eta}\left(\frac{\eta_{z}}{J}\right)\right]\right) \\
& +P_{\mathbf{U}}{ }^{-1} J\left(\frac{\partial \widehat{\mathbf{G}}_{d}}{\partial \zeta}-\left[\mathbf{E}_{d} \frac{\partial}{\partial \zeta}\left(\frac{\zeta_{x}}{J}\right)+\mathbf{F}_{d} \frac{\partial}{\partial \zeta}\left(\frac{\zeta_{y}}{J}\right)+\mathbf{G}_{d} \frac{\partial}{\partial \zeta}\left(\frac{\zeta_{z}}{J}\right)\right]\right) \\
& =P_{\mathbf{U}}{ }^{-1} J\left(\frac{\partial \widehat{\mathbf{F}}_{d}}{\partial \eta}+\frac{\partial \widehat{\mathbf{G}}_{d}}{\partial \zeta}-\left[\mathcal{A}_{d}(\eta)+\mathcal{A}_{d}(\zeta)\right]\right),
\end{aligned}
$$

Discarding the corrective terms $\mathcal{A}$ on unstructured meshes leads to critical instabilities as the imbalance induces a constant source terms on $\mathcal{L}$ at the boundary.

The set of equations presented that far has been used by Kim and Joo [46] to derive characteristic boundary conditions in generalized coordinates. Their method reads as follows for a $\xi$-normal boundary. First, Eq. (13) is used to provide an initial guess for $\mathcal{L}$ using the $\xi$-convective flux derivative extrapolated from the inner domain's solution. Second, $\mathcal{L}$ is modified using classical NSCBC conditions such as, for instance, non-reflectivity. The new $\xi$-convective characteristics are labeled $\mathcal{L}^{*}$. Third, Eq. (13) is used again to obtain the corresponding corrected $\xi$-convective flux derivative $\left(\partial_{\xi} \widehat{\mathbf{E}}_{c}\right)^{*}$. Fourth, this quantity is returned into Eq. (12) to march the solution in time. Note that in the particular case of a hard wall, entropy waves are canceled which transforms Eq. (9) into a condition on $\mathcal{D}$ instead of $\mathcal{L}$. Kim and Joo showed that the diffusive flux needs to be corrected a posteriori to properly enforce a no-penetration boundary condition, which adds an extra step.

\subsection{Compact formulation of the characteristic boundary conditions}

Given the current state of knowledge on characteristic boundary conditions, the following method is developed. The core concept is to replace the convective/diffusive characterization with a normal/tangent determination. Hence, the convective and diffusive fluxes are conveniently summed up during their evaluation as $\widehat{\mathbf{E}}=\widehat{\mathbf{E}}_{c}+\widehat{\mathbf{E}}_{d}, \widehat{\mathbf{F}}=\widehat{\mathbf{F}}_{c}+\widehat{\mathbf{F}}_{d}$ and $\widehat{\mathbf{G}}=\widehat{\mathbf{G}}_{c}+\widehat{\mathbf{G}}_{d}$ such that Eq. (12) has now further been reduced to:

$$
\frac{1}{J} \frac{\partial \mathbf{U}}{\partial t}+\frac{\partial \widehat{\mathbf{E}}}{\partial \xi}+\frac{\partial \widehat{\mathbf{F}}}{\partial \eta}+\frac{\partial \widehat{\mathbf{G}}}{\partial \zeta}=0 .
$$

Eqs. (13) and (14) are manipulated to express the sum of the flux derivatives along the $\xi$-direction (the second term in Eq. (17)) as:

$$
\frac{\partial \widehat{\mathbf{E}}}{\partial \xi}=\frac{1}{J} P_{\mathbf{U}}(\mathcal{L}+\mathcal{D})+\mathcal{A}_{c}(\xi)+\mathcal{A}_{d}(\xi)
$$

By multiplying Eq. (17) by $P_{\mathbf{U}}^{-1}$ and using Eq. (18) we have:

$$
\frac{1}{J} \frac{\partial \mathbf{W}}{\partial t}+\frac{1}{J}(\mathcal{L}+\mathcal{D})=-P_{\mathbf{U}}^{-1}\left(\frac{\partial \widehat{\mathbf{F}}}{\partial \eta}+\frac{\partial \widehat{\mathbf{G}}}{\partial \zeta}+\mathcal{A}_{c}(\xi)+\mathcal{A}_{d}(\xi)\right)
$$


The next step consists in recognizing that $\mathcal{A}_{c}(\xi)+\mathcal{A}_{d}(\xi)=-\mathcal{A}_{c}(\eta)-\mathcal{A}_{c}(\zeta)-\mathcal{A}_{d}(\eta)-\mathcal{A}_{d}(\zeta)$ thanks to Eq. (5). As a result, Eq. (19) is recast into:

$$
\begin{aligned}
\frac{1}{J} \frac{\partial \mathbf{W}}{\partial t}+\frac{1}{J}(\mathcal{L}+\mathcal{D})=-P_{\mathbf{U}}^{-1} & \left(\frac{\partial \widehat{\mathbf{F}}_{c}}{\partial \eta}+\frac{\partial \widehat{\mathbf{G}}_{c}}{\partial \zeta}-\left[\mathcal{A}_{c}(\eta)+\mathcal{A}_{c}(\zeta)\right]\right. \\
+ & \left.\frac{\partial \widehat{\mathbf{F}}_{d}}{\partial \eta}+\frac{\partial \widehat{\mathbf{G}}_{d}}{\partial \zeta}-\left[\mathcal{A}_{d}(\eta)+\mathcal{A}_{d}(\zeta)\right]\right)
\end{aligned}
$$

This last equation is equivalent to Eq. (9) thanks to Eqs. (15) and (16). Therefore, it is possible to evaluate $\mathcal{L}+\mathcal{D}$ and $\mathcal{T}_{c}+\mathcal{T}_{d}$ from the sum of the respective flux derivatives. Hence, the convective/diffusive characterization is no longer relevant. Instead, the normal (i.e. being corrected) and tangent (i.e. evaluating the correction) NSCBC components are introduced and are respectively defined as:

$$
\left\{\begin{array}{l}
\mathcal{N}=(\mathcal{L}+\mathcal{D}) / J \\
\mathcal{S}=\left(\mathcal{T}_{c}+\mathcal{T}_{d}\right) / J
\end{array}\right.
$$

To summarize, the equations used to prescribe NSCBC at a $\xi$-normal boundary are:

$$
\frac{1}{J} \frac{\partial \mathbf{W}}{\partial t}+\mathcal{N}=-\mathcal{S}
$$

with

$$
\mathcal{N}=P_{\mathbf{U}}^{-1}\left(\frac{\partial \widehat{\mathbf{E}}}{\partial \xi}-\mathcal{A}_{c}(\xi)-\mathcal{A}_{d}(\xi)\right)
$$

and

$$
\mathcal{S}=P_{\mathbf{U}}^{-1}\left(\frac{\partial \widehat{\mathbf{F}}}{\partial \eta}+\frac{\partial \widehat{\mathbf{G}}}{\partial \zeta}+\mathcal{A}_{c}(\xi)+\mathcal{A}_{d}(\xi)\right)
$$

Finally, it is sometimes more convenient to specify boundary conditions in terms of primitive variables $\mathbf{Q}=$ $(\rho, u, v, w, p)$ rather than characteristic ones. To this end, the system of equations given by Eq. (22) is recast in primitive form using the transformation matrix $P_{\mathbf{Q}}$ given in the Appendix. This reads as:

$$
\begin{aligned}
& -\frac{1}{J} \frac{\partial \rho}{\partial t}=n_{x}\left(\mathcal{N}_{1}+\mathcal{S}_{1}\right)+n_{y}\left(\mathcal{N}_{2}+\mathcal{S}_{2}\right)+n_{z}\left(\mathcal{N}_{3}+\mathcal{S}_{3}\right)+\frac{1}{2 c^{2}}\left(\mathcal{N}_{+}+\mathcal{S}_{+}+\mathcal{N}_{-}+\mathcal{S}_{-}\right) \\
& -\frac{1}{J} \frac{\partial u}{\partial t}=-n_{z}\left(\mathcal{N}_{2}+\mathcal{S}_{2}\right)+n_{y}\left(\mathcal{N}_{3}+\mathcal{S}_{3}\right)+\frac{n_{x}}{2 \rho c}\left(\mathcal{N}_{+}+\mathcal{S}_{+}-\mathcal{N}_{-}-\mathcal{S}_{-}\right) \\
& -\frac{1}{J} \frac{\partial v}{\partial t}=+n_{z}\left(\mathcal{N}_{1}+\mathcal{S}_{1}\right)-n_{x}\left(\mathcal{N}_{3}+\mathcal{S}_{3}\right)+\frac{n_{y}}{2 \rho c}\left(\mathcal{N}_{+}+\mathcal{S}_{+}-\mathcal{N}_{-}-\mathcal{S}_{-}\right) \\
& -\frac{1}{J} \frac{\partial w}{\partial t}=-n_{y}\left(\mathcal{N}_{1}+\mathcal{S}_{1}\right)+n_{x}\left(\mathcal{N}_{2}+\mathcal{S}_{2}\right)+\frac{n_{z}}{2 \rho c}\left(\mathcal{N}_{+}+\mathcal{S}_{+}-\mathcal{N}_{-}-\mathcal{S}_{-}\right) \\
& -\frac{1}{J} \frac{\partial p}{\partial t}=\frac{1}{2}\left(\mathcal{N}_{+}+\mathcal{S}_{+}+\mathcal{N}_{-}+\mathcal{S}_{-}\right)
\end{aligned}
$$

Applying characteristic boundary conditions to a face consists in modifying $\mathcal{N}$ to satisfy the prescribed behaviour. The modified normal component which verifies the desired conditions is called $\mathcal{N}^{*}$. The corresponding modified normal flux derivative is defined as 


$$
\left(\frac{\partial \widehat{\mathbf{E}}}{\partial \xi}\right)^{*}=P_{\mathbf{U}} \mathcal{N}^{*}+\mathcal{A}_{c}(\xi)+\mathcal{A}_{d}(\xi)
$$

and can be used to march the solution in time in the elements bordering the domain, now accounting for the NSCBC:

$$
\frac{1}{J} \frac{\partial \mathbf{U}}{\partial t}+\left(\frac{\partial \widehat{\mathbf{E}}}{\partial \xi}\right)^{*}+\frac{\partial \widehat{\mathbf{F}}}{\partial \eta}+\frac{\partial \widehat{\mathbf{G}}}{\partial \zeta}=0 .
$$

Note that before the NSCBC can correct the face-normal flux derivative, a first calculation of all the fluxes is needed to provide a prior evaluation of $\mathcal{N}$ and $\mathcal{S}$ at the NSCBC face. This first guess either uses the extrapolated solution or informed Dirichlet/Neumann conditions. The former is usually used for open boundaries, while the latter is more suited for hard boundaries.

To conclude this section, the main novelty pertaining to NSCBC schemes is summarized as follows. While usual implementations of viscous NSCBC $[46,47]$ treat the diffusive fluxes as a source term in the right-hand-side of Eq. (9), the formulation derived above does not discriminate between convective and diffusive fluxes. It corrects all the boundary's normal fluxes in one fell swoop. This method permits several computational shortcuts compared to the usual implementation. First, there is no need to store both convective and diffusive fluxes which halves the memory allocation cost. Second, the computation and extrapolation of the normal and tangent flux derivatives to the boundary faces are also halved. Third, the method now always accounts for diffusivity and corrects it for no added coding complexity, as opposed to the original method [46] which requires an a posteriori correction of the normal diffusive flux derivative to enforce a true hard boundary. Finally, both methods are mathematically identical as summing or splitting the fluxes merely redistributes terms within the same system of equations, i.e. Eq. (9) is equivalent to Eq. (22).

\section{Sub-classes of NSCBC}

For the sake of completeness, various sub-classes of NSCBC are presented in this section. Without loss of generality, a $\xi$-normal boundary is once again considered to illustrate them. The equations given for each NSCBC permit to derive $\mathcal{N}^{*}$, which in turn allows to evaluate the corrected normal flux derivative at the boundary. First, typical non-reflecting open boundary conditions are presented, both in subsonic inflow and outflow configurations. Then, hard wall and impedance boundary conditions are presented. The latter represents a complex sub-class of NSCBC where the surface's reflectivity depends on the incident wave's frequency.

\subsection{Non-reflecting boundary conditions}

\subsubsection{Subsonic outflow}

For a subsonic outflow, the $\left(u_{n}-c\right)$-traveling characteristic (i.e. the backwards acoustic wave) enters the computational domain, while the four others exit it. One thermodynamic property needs to be enforced to obtain a numerically well-behaved boundary condition [17]. Further, in order to avoid spurious reflections at the boundary, the entering acoustic characteristic $\partial_{t} W_{-}$can be canceled. This theoretically results in a well-behaved and perfectly non-reflective conditions in the ideal case of a wave normally-incident to the boundary. In order to satisfy $\partial_{t} W_{-}=0$, Eq. (22) prescribes:

$$
\mathcal{N}_{-}^{*}=-\mathcal{S}_{-} .
$$

This classical non-reflecting pressure outflow NSCBC has been the focus of many studies, and many empirical modifications to Eq. (28) have been suggested. For instance, Poinsot and Lele suggested to use a pressure relaxation rate $K_{p}\left[\mathrm{~s}^{-1}\right]$ and target back-pressure $p_{\infty}$ such that $\partial_{t} W_{-}=K_{p}\left(p_{\infty}-p\right)$ rather than imposing $W_{-}=0$. Failure to do so eventually results in a pressure drift in time as the computational domain receives no pressure feedback from the outer world. Note that the stiffness caused by the relaxation term induces some acoustic reflection, which characterizes the NSCBC as a partially non-reflecting characteristic boundary condition.

Further, Yoo et al. [48] developed Eq. (28) by accounting for the transverse terms (i.e the tangent fluxes), which considerably improved the behavior when the outflow exhibits strong inhomogeneities (such as a vortex). They observed that accounting for the whole tangent flux at high Mach number increased the boundary condition's reflectivity, 
and derived a formulation which partly relaxes around the exact tangent flux $\mathcal{T}_{c,-}^{\text {exact }}$. It reads, using the usual Eulerian $\left(\mathcal{L}, \mathcal{T}_{c}\right)$-determination, as:

$$
\frac{1}{J} \partial_{t} W_{-}+\mathcal{L}_{-}^{*}+\alpha\left(\mathcal{T}_{c,-}-\mathcal{T}_{c,-}^{\text {exact }}\right)=0
$$

where $\alpha$ is the relaxation constant which, in the low-Mach limit, tends towards the bulk outflow Mach number. The final expression from [48] for the non-reflecting characteristic boundary condition is obtained by replacing $W_{-}$with $=K_{p}\left(p_{\infty}-p\right)$ in Eq. (29):

$$
\mathcal{L}_{-}^{*}=\frac{1}{J} K_{p}\left(p-p_{\infty}\right)+\alpha \mathcal{T}_{c,-}^{\text {exact }}-(1-\alpha) \mathcal{T}_{c,-}
$$

Yoo and Im [47] later added the contribution of the diffusive fluxes with no particular treatment. The current formulation naturally includes them by using the compact $(\mathcal{N}, \mathcal{S})$ determination from the start. Finally, the modified $\mathcal{N}_{-}^{*}$ is evaluated for a characteristic pressure outflow condition as:

$$
\mathcal{N}_{-}^{*}=\frac{1}{J} K_{p}\left(p-p_{\infty}\right)+\alpha \mathcal{S}_{-}^{\text {exact }}-(1-\alpha) \mathcal{S}_{-}
$$

There is no clear consensus yet at to what the value $\alpha$ should be. The averaged bulk Mach number at the whole boundary and the local instantaneous Mach number are the simplest and most common choices, with the former usually performing best $[47,49,50]$. Liu and Vassiliev [51] improved the method by calculating a damping coefficient based on the streamline incident angle with the boundary's normal, and by only relaxing certain components of the transverse flux.

\subsubsection{Subsonic inflow}

For a subsonic inflow, the $\left(u_{n}-c\right)$-traveling characteristic exits the computational domain, while the four others enter it. Four thermodynamic properties need to be enforced to obtain a numerically well-behaved boundary condition [17]. In this case, it is convenient to work with primitive variables and Eqs. (25). An equation on $\partial_{t} T$ can be derived using the ideal gas law. Finally, the following system is adapted from the work of Yoo et al. [48] to the new formalism in order to evaluate the corrected $\mathcal{N}^{*}$ :

$$
\begin{aligned}
& \frac{1}{J}\left[K_{u}\left(u-u_{\infty}\right)-\frac{\partial u}{\partial t}\right]=-n_{z}\left(\mathcal{N}_{2}^{*}+\mathcal{S}_{2}\right)+n_{y}\left(\mathcal{N}_{3}^{*}+\mathcal{S}_{3}\right)+\frac{n_{x}}{2 \rho c}\left(\mathcal{N}_{+}^{*}+\mathcal{S}_{+}-\mathcal{N}_{-}-\mathcal{S}_{-}\right) \\
& \frac{1}{J}\left[K_{v}\left(v-v_{\infty}\right)-\frac{\partial v}{\partial t}\right]=n_{z}\left(\mathcal{N}_{1}^{*}+\mathcal{S}_{1}\right)-n_{x}\left(\mathcal{N}_{3}^{*}+\mathcal{S}_{3}\right)+\frac{n_{y}}{2 \rho c}\left(\mathcal{N}_{+}^{*}+\mathcal{S}_{+}-\mathcal{N}_{-}-\mathcal{S}_{-}\right) \\
& \frac{1}{J}\left[K_{w}\left(w-w_{\infty}\right)-\frac{\partial w}{\partial t}\right]=-n_{y}\left(\mathcal{N}_{1}^{*}+\mathcal{S}_{1}\right)+n_{x}\left(\mathcal{N}_{2}^{*}+\mathcal{S}_{2}\right)+\frac{n_{z}}{2 \rho c}\left(\mathcal{N}_{+}^{*}+\mathcal{S}_{+}-\mathcal{N}_{-}-\mathcal{S}_{-}\right) \\
& \frac{1}{J}\left[K_{T}\left(T-T_{\infty}\right)-\frac{\rho}{T} \frac{\partial T}{\partial t}\right]=-n_{x}\left(\mathcal{N}_{1}^{*}+\mathcal{S}_{1}\right)-n_{y}\left(\mathcal{N}_{2}^{*}+\mathcal{S}_{2}\right)-n_{z}\left(\mathcal{N}_{3}^{*}+\mathcal{S}_{3}\right) \\
&+\frac{(\gamma-1)}{2 c^{2}}\left(\mathcal{N}_{+}^{*}+\mathcal{S}_{+}-\mathcal{N}_{-}-\mathcal{S}_{-}\right)
\end{aligned}
$$

where $\left(u_{\infty}, v_{\infty}, w_{\infty}, T_{\infty}\right)$ are the prescribed inflow primitive variables and $\left(K_{u}, K_{v}, K_{w}, K_{T}\right)$ their associated relaxation rates. $\gamma$ is the gas heat specific ratio. If known time-varying inflow conditions are prescribed, the relaxation terms in the left hand side of these equations can be dropped, which recovers the exact form of the primitive equations (Eqs. (25)). Otherwise, the time-derivatives are discarded and empirical relaxation rates have to be specified for each prescribed variable to prevent a numerical drift from the inflow values, just as with a partially non-reflecting outlet. In this system of equations, all the $\mathcal{S}$ and $\mathcal{N}_{-}$can be evaluated from the inner domain's solution, yielding a system of 4 equations and 4 unknown. It has been observed by Yoo et al. [48] that the transverse terms are generally not necessary due to most variables being prescribed. Last, it is possible to impose total quantities through characteristics instead, as done in [52]. 


\subsection{Time-domain impedance boundary conditions}

As a sub-class of NSCBC, a TDIBC is also applied through the flux balance correction (Eq. (26)). Its implementation is detailed below. Notably, it generalizes the work of Monteghetti et al. [53, 54] from the linearized Euler equations (LEE) to the Navier-Stokes equations.

In order to capture the broadband response of an acoustically-reacting surface in the time-domain, the TDIBC is comprised of three elements [54]: 1) a model of the surface's impedance in Laplace-space, 2) a discretization scheme in the time-domain of the model and finally, 3) coupling with the Navier-Stokes equations. These three components are presented subsequently, preceded by a brief presentation of useful notations and concepts pertaining to linear acoustics. For clarity purposes, the tilde superscript denotes throughout the paper an imaginary quantity and/or a function of the Laplace-variable $s=j \omega$.

\subsubsection{Acoustic impedance for linearized Euler equations}

In acoustics, the impedance $\widetilde{z}$ of a medium characterizes its reaction to a pressure perturbation. For a medium where the impedance depends only on the perturbation's angular frequency $\omega$ and its location in space $\mathbf{x}$, it can be defined as:

$$
\widetilde{z}(\mathbf{x}, s)=\frac{\widetilde{p}(\mathbf{x}, s)}{\widetilde{u}_{n}(\mathbf{x}, s)}
$$

where $\widetilde{p}$ and $\widetilde{u}_{n}$ are the Laplace-transformed acoustic pressure and longitudinal velocity perturbations along the wavefront's normal $\mathbf{n}$. The impedance possesses a real-part called the resistance and an imaginary part called the reactance which have units of $\mathrm{kg} \cdot \mathrm{m}^{2} / \mathrm{s}$.

A reflection coefficient $\widetilde{\beta}$ is derived from the impedance as:

$$
\widetilde{\beta}=\frac{\widetilde{z}-z_{0}}{\widetilde{z}+z_{0}} .
$$

where the medium's reference impedance $z_{0}$ equals to $\rho_{0} c_{0}$. The inverse-Laplace transform of $\widetilde{z}$ and $\widetilde{\beta}$ are respectively called $z$ and $\beta$. Let us define in the real time-domain the pressure and longitudinal velocity fluctuations $p^{\prime}$ and $u_{n}^{\prime}$. For the LEE, the reflected and incident wave amplitudes are respectively $v_{+}^{\prime}=p^{\prime}-\rho_{0} c_{0} u_{n}^{\prime}$ and $v_{-}^{\prime}=p^{\prime}+\rho_{0} c_{0} u_{n}^{\prime}$. They correspond to the right and left running acoustic characteristics traveling at the medium's speed of sound $c_{0}$. Finally, the time-domain impedance $\mathcal{Z}$ and scattering $\mathcal{B}$ operators respectively define the following positive-real functions:

$$
p^{\prime}(t)=\left[z \star u_{n}^{\prime}\right](t)=\mathcal{Z}\left[u_{n}^{\prime}\right](t)
$$

and

$$
v_{+}^{\prime}(t)=\left[\beta \star v_{-}^{\prime}\right](t)=\mathcal{B}\left[v_{-}^{\prime}\right](t)
$$

where $\star$ denotes the time-domain convolution product operator between real functions. Both $\mathcal{Z}$ and $\mathcal{B}$ define a TDIBC. However, as suggested first by Brambley and Gabard [55] and later formally demonstrated by Monteghetti [54], adopting a $\mathcal{B}$-formalism rather than a $\mathcal{Z}$-formalism yields a more stable TDIBC due to $\widetilde{\beta}$ being bounded contrary to $\bar{z}$. This proved to relax the restriction on the Courant-Friedrichs-Lewy number [54] which is of paramount importance to CFD applications. The reflection coefficient's admissibility conditions, defined by Rienstra [56] and later consolidated in [54], ensure a stable and physically-relevant TDIBC implementation.

\subsubsection{Delayed multi-pole representation of a surface's impedance}

As explained in [54], the first component of a TDIBC consists in an impedance model which relates the boundary's pressure fluctuations to the normal velocity fluctuations. In the present study, the acoustically absorbent surface is simply described as a densed set of narrow cavities with rigid ends, each represented as an acoustic resonator. The whole structure forms a honeycomb which impedance $\widetilde{z}$ is modeled as in Jones et al. [57].

Once an impedance model has been chosen, Eq. (34) is used to evaluate the corresponding reflection coefficient $\widetilde{\beta}$. The second component of a TDIBC consists in the discrete representation of the reflection coefficient in the timedomain. This amounts to constructing the operator $\mathcal{B}(t)$ from $\widetilde{\beta}(s)$. The Oscillo-Diffusive Representation (ODR) from 
[53] is used to this end. The reader is referred to these papers to obtain a detailed presentation of the ODR of irrational functions in Laplace space. The ODR provides the following approximated discrete representation for $\widetilde{\beta}$ :

$$
\widetilde{\beta}(s)=\beta_{\infty}+\sum_{n=1}^{N_{s}} \frac{\widetilde{r}_{1, n}}{s-\widetilde{s}_{n}}+\sum_{k=1}^{N_{v}} \frac{\mu_{1, k}}{s+v_{k}}+e^{-s \tau}\left(\sum_{n=1}^{N_{s}} \frac{\widetilde{r}_{2, n}}{s-\widetilde{s}_{n}}+\sum_{k=1}^{N_{v}} \frac{\mu_{2, k}}{s+v_{k}}\right)
$$

It is comprised of a finite number of $N_{s}$ complex oscillatory poles $\widetilde{\mathbf{r}}$ (each weightened by a factor $\widetilde{\mathbf{s}}$ ) and of $N_{\widetilde{\phi}}$ real diffusive poles $\boldsymbol{v}$ (and corresponding weights $\boldsymbol{\mu}$ ). The delay operator $e^{-\tau s}$ corresponds to the back-and-forth traveling time of a wave inside the liner's cavity. Importantly, the ODR offers the possibility to optimize the poles and weights to match any given impedance law. The underlying interest of the ODR-based discretization of $\beta(s)$ lies in the transformation of the convolution product (Eq. (36)) into a sum of complex auxiliary functions solutions of ordinary differential equations (ODE), as demonstrated by Monteghetti et al. [53]. This powerful result permits to represent the scattering operator $\mathcal{B}$ (i.e. the TDIBC) with ODE which can use the same time-marching scheme as for the flow conservative variables. The evaluation of $\mathcal{B}$ uses as many auxiliary functions as the number of poles used in the ODR of $\widetilde{\beta}$. It reads as:

$$
\mathcal{B}(t)=\beta_{\infty} v_{-}^{\prime}(t)+\sum_{n=1}^{N_{s}} \widetilde{r}_{1, n} \widetilde{\phi}\left(t,-\widetilde{s}_{n}\right)+\sum_{k=1}^{N_{v}} \mu_{1, k} \widetilde{\phi}\left(t, v_{k}\right)+\sum_{n \in I} \widetilde{r}_{2, n} \widetilde{\phi}\left(t-\tau,-\widetilde{s}_{n}\right)+\sum_{k=1}^{N_{v}} \mu_{2, k} \widetilde{\phi}\left(t-\tau, v_{k}\right)
$$

where the $\widetilde{\phi}$ are evaluated using:

$$
\left\{\begin{array}{l}
\partial_{t} \widetilde{\phi}(t, s)=-s \widetilde{\phi}(t, s)+v_{-}^{\prime}(t) \quad(t>0, s \in \mathbb{C}) \\
\widetilde{\phi}(t, 0)=\widetilde{\phi}(0, s)=0
\end{array}\right.
$$

where $s$ is replaced by all the diffusive and oscillatory poles.

The $e^{-\tau s}$ operator can be resolved in different ways. In the time-domain, its inverse-laplace transform simply consists of a delay $\tau$. Following [54], another set of auxiliary functions $\widetilde{\psi}$ are defined as:

$$
\widetilde{\psi}\left(t, s, l_{\tau}\right)=\widetilde{\phi}(t-\tau, s) .
$$

The delay is resolved by transporting at the speed of sound at the TDIBC, $c_{\tau}$, the $\widetilde{\phi}$ variables over another spatial coordinate $\theta$ of length equal to $\tau / c_{\tau}$. The additional auxiliary functions $\widetilde{\psi}$ are solutions of the following transport equation:

$$
\left\{\begin{array}{l}
\partial_{t} \widetilde{\psi}(t, s, \theta)=c_{\tau} \partial_{\theta} \widetilde{\psi}(t, s, \theta) \quad\left(t>0, \theta \in\left[0, l_{\tau}\right], s \in \mathbb{C}\right) \\
\widetilde{\psi}(t, s, 0)=\widetilde{\phi}(t, s) \\
\widetilde{\psi}(0, s, \theta)=0
\end{array}\right.
$$

This amounts to resolving an advection problem for each pole at each flux points located on a TDIBC face. A number of elements $N_{e}$ is chosen to discretize the $\theta$-space. Its length $l_{\tau}$, maximum delay $\tau$ and advection speed $c_{\tau}$ are related as $\tau=l_{\tau} / c_{\tau}$. The number of elements per wavelength (EPW) determines the cut-off frequency $f_{\max }=$ $\left(N_{e} \times \mathrm{EPW}\right) /(2 \tau)$ with a minimum of EPW $=2$ needed to avoid aliasing. It is remembered that the ODR is not unconditionally passive outside the $v$-bounds. Therefore, it is suggested to choose $f_{\max } \leq \max (|v|)$ to naturally filter out higher frequencies.

The advection problem flux derivative $c_{\tau} \partial_{\theta} \widetilde{\psi}(t, s, \theta)$ is typically calculated using a $6^{\text {th }}$-order SD method. In order to mitigate the computational cost, a single $q$-contiguous data array encompassing all the $\widetilde{\psi}$ variables at all the solution and flux points inside each $\theta$-element is created. Hence, a single call to the extrapolation and flux derivation routines is required per each sub-iteration to update all of them at once. This permits to make an optimal use of the CPU cache memory and alleviate as much as possible the computational cost of this method. The advection speed $c_{\tau}$ is constant along $\theta$ and equals the speed of sound at the isothermal wall temperature. At each boundary between the $N_{e}$ elements along $\theta$, a common flux is chosen through simple upwinding. The $q$-contiguous array also permits to resolve all the Riemann problems associated to a TDIBC face at once. The boundary conditions simply consist in using the variables $\widetilde{\phi}$ as inflow and sending the outflow into the variables $\widetilde{\psi}$. Finally, the auxiliary variables $\widetilde{\phi}$ and $\widetilde{\psi}$ can conveniently be 
integrated in time using the same time-marching scheme as the main simulation, which further reduces the impact on computational performance.

Alternatively, Douasbin et al. [58] showed that the TDIBC's time-delay could be resolved using a multi-pole representation of $e^{-\tau s}$. This amounts to choosing an arbitrary number of complex poles and weights optimized to resolve a pure phase shift. While Douasbin et al. showed their method was virtually loss-less and reduced the cost in memory allocation, it is prone to instabilities due to the passivity conditions being violated when using too few poles. To avoid instabilities, they applied a low-pass filter on top of the multi-pole model. Conversely, the resolution of advection equations with the SD method is inherently dissipative: the $\theta$-mesh cut-off acts as a natural filter. Tests which consisted in convecting a sinusoidal over one wavelength using $8 \mathrm{EPW}$ or more resulted in energy losses below $0.01 \%$.

\subsubsection{Coupling with Navier-Stokes equations through characteristics}

The third and last component of a TDIBC is its coupling with the flow solver, i.e. a semi-discrete scheme embedded within the NSCBC equations. A formulation derived from the linerarized Euler equations is often used, which consists in relating the pressure perturbations to the wall normal velocity. The issues arising from using such terminology are two-fold.

First, the concept of fluctuation is ill-suited as the time-averaged solution is not a priori known. Conservation of mass inside the semi-closed cavities spaning porous coating ensures that the mean wall normal velocity converges towards zero in time (yielding $\rho_{0} u_{n}=\rho_{0} u_{n}^{\prime}$ ). Yet, it is unclear how $p^{\prime}$ is evaluated. While it would be possible to evaluate $p^{\prime}$ from $u_{n}^{\prime}$ by using a $\widetilde{z}$-based TDIBC, $\widetilde{\beta}$-based TDIBC are much preferable as explained by [54]. In this case, the TDIBC input consists of a characteristic containing unknown pressure fluctuations (Eq. (36)). A similar formulation based on the wall softness (which equals $\mathbb{1}+\mathcal{B}$ ) was used by [59, 24] to derive TDIBC for NSE. The incident linearized characteristic was estimated through a first-order Taylor expansion in space based on the velocity and pressure gradients at the wall. The fluctuating wall pressure/velocity were then evaluated as averaged/differences of the incident and reflected linearized characteristics, as used by Fung and Ju [60], and imposed as Dirichlet conditions.

Second, the treatment of the diffusive flux at the impedance boundary is an important aspect of the derivation of a TDIBC compatible with the NSE. However, to the authors' best knowledge, it has not been addressed yet. In the case where the TDIBC is imposed through the LEE' characteristics, diffusion has effectively been decoupled.

In order to avoid these shortcomings, the TDIBC are now derived in the Navier-Stokes framework. The conservation equations for the acoustic characteristics, introduced in Sec. 2.2, are Eqs. (10)d and (10)e. They serve as the knot between the NSCBC and TDIBC formulations. It is reminded that $\partial W_{+}$and $\partial W_{-}$respectively define the Navier-Stokes $\left(u_{n}+c\right)$-traveling incident and the $\left(u_{n}-c\right)$-traveling reflected characteristics (Eq. (10)). They read as:

$$
\left\{\begin{array}{l}
\partial W_{-}=\partial p-\rho c \partial u_{n} \\
\partial W_{+}=\partial p+\rho c \partial u_{n}
\end{array}\right.
$$

The discrete scattering operator $\mathcal{B}$ is evaluated in time and space by Eq (38). $\mathcal{B}$ yields the TDIBC given in Eq. (36) valid for LEE only. As $\mathcal{B}$ is a linear function of $v_{-}^{\prime}$, it follows from Eq. (36) that:

$$
\partial_{t} W_{-}=\mathcal{B}\left[\partial_{t} W_{+}\right]
$$

which, thanks to Eq. (22), is reorganized into:

$$
\mathcal{N}_{-}+\mathcal{S}_{-}=\mathcal{B}\left[\mathcal{N}_{+}+\mathcal{S}_{+}\right]
$$

Finally, the coupling with the NSCBC method presented in Sec. 2 is operated by correcting the normal component pertaining to the incident acoustic characteristic, $\mathcal{N}_{-}^{*}$. It follows from Eq. (44) that:

$$
\mathcal{N}_{-}^{*}=\mathcal{B}\left[\mathcal{N}_{+}+\mathcal{S}_{+}\right]-\mathcal{S}_{-}
$$

The scattering operator is then comprised of a weighted sum of auxiliary functions $\widetilde{\phi}$ and $\widetilde{\psi}$ evaluated with Eqs. (39) and (41) where $v_{-}^{\prime}$ has been replaced by $\left(\mathcal{N}_{+}+\mathcal{S}_{+}\right)$. Then, as with any other NSCBC presented in this study, Eq. (26) is used to evaluate the corrected normal flux. Note that the treatment of diffusivity has been implicitly addressed by considering the compact formulation for NSCBC given in Sec. 2.2 (i.e. using $\mathcal{N}=\mathcal{L}+\mathcal{D}$ ). Obviously, had $\mathcal{D}$ 
been discarded from the TDIBC, a component partially responsible for the wall-normal velocity and pressure gradient would have been neglected, leading to a wrong estimation of the incident and reflected acoustic characteristics.

Finally, this coupling method injects the TDIBC by adjusting the flux balance at the boundary through Eq. (26). Therefore, a prior estimate of the flux derivatives at the boundary is needed, just as with the non-reflecting conditions for open boundaries presented above. In Navier-Stokes flows, the TDIBC is considered as an isothermal no-slip surface. Hence, Dirichlet conditions $(u, v, w, T)=\left(u . n_{x}, v . n_{y}, w . n_{z}, T_{w}\right)$ are first applied at the boundary to provide an informed prior estimation of the flux derivatives. This ensures that the tangent component $\mathcal{S}$ and diffusive fluxes accounts for the no-slip boundary condition (which is tangential by nature) and that the correct speed of sound is evaluated. The wall-normal velocity and pressure are not explicitly specified: only the tangent component is set to zero. Note that imposing $\mathcal{N}_{i}^{*}=-\mathcal{S}_{i}$ for $i \in[1,2,3]$ as in $[17,46]$ would only impose null time-derivatives on the velocities (see Eqs. (25)) at the face where the solution is not stored. This would only weakly imposes the desired boundary conditions, and was observed to be a source of instability. Likewise, if the initial computation of the normal and tangent flux derivatives uses extrapolated values (as with open boundaries) instead of Dirichlet conditions, the no-slip condition is only weakly enforced and subject to numerical drift. As a result, a Navier-Stokes-based TDIBC uses partial Dirichlet conditions before the normal-flux' correction. Conversely, a Euler-based TDIBC simply uses the extrapolated conservative variables to evaluate the flux derivatives.

\section{Implementation of NSCBC with strong discontinuous spectral methods}

Section 2 detailed the derivation of the classical NSCBC in strong conservative form for multi-dimensional generalized coordinates, laying the ground for an application within the framework of strong discontinuous spectral methods. The system of equations governing various types of NSCBC have been presented in Sec. 3 and all rely on the application of Eq. (26) at the boundary. Implementation algorithms for NSCBC with both the SD and FR method are now presented. First, the application with the SD method is presented. Later, it is shown that NSCBC can similarly be implemented with the family of FR schemes.

\subsection{NSCBC with the spectral difference method}

\subsubsection{The SD scheme}

The SD method is first presented for an order of accuracy $q+1$ to introduce notations and provide context for the presentation of the NSCBC implementation. A one-dimensional domain is considered for clarity purposes without loss of generality. The governing equation reads as:

$$
\frac{1}{J} \frac{\partial \mathbf{U}}{\partial t}=-\frac{\partial \widehat{\mathbf{E}}}{\partial \xi}
$$

where $\widehat{\mathbf{E}}=\widehat{\mathbf{E}}_{c}+\widehat{\mathbf{E}}_{d}=\mathbf{E}_{c} \frac{\xi_{x}}{J}+\mathbf{E}_{d} \frac{\xi_{x}}{J}$ is reminded to account for both diffusive and convective fluxes.

During initialization, each element is further discretized into a standardized [0,1] domain. Then, $q+1$ solution points and $q+2$ flux points are distributed inside the element using respectively the Gauss and Legendre quadrature points as in [31], though other geometric distributions exist. Flux points are also placed at the element boundaries at 0 and 1 in order to encompass all the solution points and ensure communication with neighboring elements. These points are respectively defined by their standardized coordinate vectors $\mathbf{S P}_{l \in[1, q+1]}$ and $\mathbf{F} \mathbf{P}_{l \in[1, q+2]}$. The conservative variables are stored at all the solution points, where they are marched in time through Eq. (46), while the flux points form a geometrical support used to reconstruct a continuous flux in the form of a $(q+1)$-order Lagrange polynomial. Let us call $\mathbf{L}_{l, \mathrm{SP}}(\xi)$ (respectively $\mathbf{L}_{l, \mathrm{FP}}(\xi)$ ) the Lagrange polynomial of degree $q$ (respectively $q+1$ ) based on solution (respectively flux) point of index $l$.

At a particular time, the solution vector $\mathbf{U}_{l}$ with $l \in[1, q+1]$ contains the conservative variables stored at each solution point. It is used to construct a $q$-order polynomial representation of a continuous solution $\overline{\mathbf{U}}(\xi)$ across the element:

$$
\overline{\mathbf{U}}(\xi)=\sum_{l=1}^{q+1}\left[\mathbf{U}_{l} \mathbf{L}_{l, \mathrm{SP}}(\xi)\right], \quad \xi \in[0,1]
$$


This polynomial is then used to interpolate/extrapolate the conservative variables at all the flux points and evaluate the (q+2)-long flux vector $\widehat{\mathbf{E}}\left(\overline{\mathbf{U}}\left(\mathbf{F P}_{l \in[1, q+2]}\right)\right)$, or more simply referred to as $\widehat{\mathbf{E}}_{l \in[1, q+2]}$. A Riemann problem is solved at the element boundaries which provides element-to-element communication and ensures flux continuity across the whole computational domain. Once the boundary fluxes have been updated, a $(q+1)$-order Lagrange polynomial is reconstructed from the flux points:

$$
\overline{\mathbf{E}}(\xi)=\sum_{l=1}^{q+2}\left[\widehat{\mathbf{E}}_{l} \mathbf{L}_{l, \mathrm{FP}}(\xi)\right], \quad \xi \in[0,1]
$$

The flux polynomial $\overline{\mathbf{E}}(\xi)$ is then derived in space and evaluated at the solution points:

$$
\left(\frac{\partial \widehat{\mathbf{E}}}{\partial \xi}\right)_{\mathbf{S P}}=\frac{\partial \overline{\mathbf{E}}}{\partial \xi}(\mathbf{S P})=\sum_{l=1}^{q+2}\left[\widehat{\mathbf{E}}_{l} \frac{\partial \mathbf{L}_{l, \mathrm{FP}}}{\partial \xi}(\mathbf{S P})\right]
$$

which permits to march the solution in time through Eq. (46). This last step completes the semi-discrete formulation of the SD method. The method is easily extended to higher dimensions in generalized coordinates. Finally, the method is globally discontinuous in terms of conservative variables while retaining a globally continuous flux.

\subsubsection{Semi-discrete algorithm of NSCBC for $S D$}

The implementation algorithm of NSCBC for a $(q+1)$-order SD method is now presented. For the sake of simplicity, let us consider the same one-dimensional configuration and notations as before. The NSCBC is arbitrarily placed at the element's $(\xi=1)$-boundary. The generalization to higher dimensions is straightforward for hexaheadral elements as NSCBC are applied along one dimension at a time. Work on NSCBC with tetrahedral elements is the subject of ongoing research and is not addressed in this study.

In SD, Eq. (49) is meant to be used to evaluate the derivative of the flux at the solution points. Coincidentally, the Lagrange polynomial also provides a high-order extrapolation estimate to the boundary's flux points. Hence, given that $\left(\partial_{\xi} \widehat{\mathbf{E}}\right)_{\mathbf{F P}_{q+2}}=\partial_{\xi} \overline{\mathbf{E}}(1)$, Eqs. (23) and (24) are used to evaluate $\mathcal{N}$ and $\mathcal{S}$ at the face. Next, $\mathcal{N}^{*}$ is calculated from the specified NSCBC (see Sec. 3). Finally, Eq. (26) is used to obtain the corrected flux derivative $\left(\partial_{\xi} \widehat{\mathbf{E}}\right)_{\mathbf{F P}_{q+2}^{*}}^{*}$ at the face's flux points. This correction now somehow needs to feedback into $\left(\partial_{\xi} \widehat{\mathbf{E}}\right)_{\mathbf{S P}}$ which is used to march the solution in time. Hence, the flux polynomial and its derivative must be re-evaluated from $\left(\partial_{\xi} \widehat{\mathbf{E}}\right)_{\mathbf{F P}_{q+2}}$. Two methods are derived below. The first one is computationally more expensive while the second one is faster at the expense of a greater memory usage. Both initially take advantage of the linearity of the derivative operator. First, let us recall that:

$$
\frac{\partial \overline{\mathbf{E}}}{\partial \xi}(\xi)=\sum_{l=1}^{q+2}\left[\widehat{\mathbf{E}}_{l} \frac{\partial \mathbf{L}_{l, \mathrm{FP}}}{\partial \xi}(\xi)\right]
$$

Let us now introduce a reference Lagrange polynomial $\overline{\mathbf{E}}_{0}$ constructed on the flux points which replaces $\widehat{\mathbf{E}}_{q+2}$ with zero. The three polynoms $\overline{\mathbf{E}}, \overline{\mathbf{E}}^{*}$, and $\overline{\mathbf{E}}_{0}$ verify at $\xi=1$ :

$$
\left\{\begin{array}{l}
\partial_{\xi} \overline{\mathbf{E}}(1)=\sum_{l=1}^{q+1}\left[\widehat{\mathbf{E}}_{l} \partial_{\xi} \mathbf{L}_{l, \mathrm{FP}}(1)\right]+\widehat{\mathbf{E}}_{q+2} \partial_{\xi} \mathbf{L}_{q+2, \mathrm{FP}}(1) \\
\partial_{\xi} \overline{\mathbf{E}}^{*}(1)=\sum_{l=1}^{q+1}\left[\widehat{\mathbf{E}}_{l} \partial_{\xi} \mathbf{L}_{l, \mathrm{FP}}(1)\right]+\widehat{\mathbf{E}}_{q+2}^{*} \partial_{\xi} \mathbf{L}_{q+2, \mathrm{FP}}(1) \\
\partial_{\xi} \overline{\mathbf{E}}_{0}(1)=\sum_{l=1}^{q+1}\left[\widehat{\mathbf{E}}_{l} \partial_{\xi} \mathbf{L}_{l, \mathrm{FP}}(1)\right]
\end{array}\right.
$$

Hence, we have:

$$
\left\{\begin{array}{l}
\partial_{\xi} \overline{\mathbf{E}}(1)=\partial_{\xi} \overline{\mathbf{E}}_{0}(1)+\widehat{\mathbf{E}}_{q+2} \partial_{\xi} \mathbf{L}_{q+2, \mathrm{FP}}(1) \\
\partial_{\xi} \overline{\mathbf{E}}^{*}(1)=\partial_{\xi} \overline{\mathbf{E}}_{0}(1)+\widehat{\mathbf{E}}_{q+2}^{*} \partial_{\xi} \mathbf{L}_{q+2, \mathrm{FP}}(1) .
\end{array}\right.
$$

Let us recall that $\left(\partial_{\xi} \widehat{\mathbf{E}}\right)_{q+2}^{*}=\partial_{\xi} \overline{\mathbf{E}}^{*}(1)$ and $\left(\partial_{\xi} \widehat{\mathbf{E}}\right)_{q+2}=\partial_{\xi} \overline{\mathbf{E}}(1)$. The corrected flux at the $(\xi=1)$-boundary is evaluated as: 


$$
\widehat{\mathbf{E}}_{q+2}^{*}=\widehat{\mathbf{E}}_{q+2}\left[\frac{\partial_{\xi} \overline{\mathbf{E}}^{*}(1)-\partial_{\xi} \overline{\mathbf{E}}_{0}(1)}{\partial_{\xi} \overline{\mathbf{E}}(1)-\partial_{\xi} \overline{\mathbf{E}}_{0}(1)}\right]=\widehat{\mathbf{E}}_{q+2}\left[\frac{\left.\partial_{\xi} \widehat{\mathbf{E}}\right)_{q+2}^{*}-\partial_{\xi} \overline{\mathbf{E}}_{0}(1)}{\left(\partial_{\xi} \widehat{\mathbf{E}}\right)_{q+2}-\partial_{\xi} \overline{\mathbf{E}}_{0}(1)}\right]
$$

The corrected flux vector is $\widehat{\mathbf{E}}^{*}=\left[\widehat{\mathbf{E}}_{l \in[1, q+1]}, \widehat{\mathbf{E}}_{q+2}^{*}\right]$. The last steps consist in building a corrected flux derivative Lagrange polynomial $\left(\partial_{\xi} \overline{\mathbf{E}}\right)^{*}$ and evaluating it at the solution points with Eq. (49) to march the solution in time using Eq. (27).

Alternatively, there exists a more direct way of applying the flux balance correction. $\partial_{\xi} \mathbf{L}_{l, \mathrm{FP}}$ could be evaluated during the initialization phase of the simulation and stored. This permits to bypass the need to evaluate the reference flux polynomial $\overline{\mathbf{E}}_{0}$ which alleviates the computational cost at the expense of a greater memory usage. Indeed, if $\partial_{\xi} \mathbf{L}_{q+2, \mathrm{FP}}$ is known then Eq. (52) can be used to compute the corrected flux at the ( $\left.\xi=1\right)$-boundary as:

$$
\widehat{\mathbf{E}}_{q+2}^{*}=\widehat{\mathbf{E}}_{q+2}+\left[\frac{\left(\partial_{\xi} \widehat{\mathbf{E}}\right)_{q+2}^{*}-\left(\partial_{\xi} \widehat{\mathbf{E}}\right)_{q+2}}{\partial_{\xi} \mathbf{L}_{q+2, \mathrm{FP}}(1)}\right]
$$

which permits to directly correct evaluation of the flux derivative at the solution points:

$$
\begin{aligned}
\left(\frac{\partial \widehat{\mathbf{E}}}{\partial \xi}\right)_{\mathbf{S P}}^{*} & =\left(\frac{\partial \widehat{\mathbf{E}}}{\partial \xi}\right)_{\mathbf{S P}}+\left[\widehat{\mathbf{E}}_{q+2}^{*}-\widehat{\mathbf{E}}_{q+2}\right] \partial_{\xi} \mathbf{L}_{q+2, \mathrm{FP}}(\mathbf{S P}) \\
& =\left(\frac{\partial \widehat{\mathbf{E}}}{\partial \xi}\right)_{\mathbf{S P}}+\left[\left(\frac{\partial \widehat{\mathbf{E}}}{\partial \xi}\right)_{q+2}^{*}-\left(\frac{\partial \widehat{\mathbf{E}}}{\partial \xi}\right)_{q+2}\right]\left[\frac{\partial_{\xi} \mathbf{L}_{q+2, \mathrm{FP}}(\mathbf{S P})}{\partial_{\xi} \mathbf{L}_{q+2, \mathrm{FP}}(1)}\right]
\end{aligned}
$$

To summarize, the semi-discrete NSCBC scheme for SD consists in the following steps applied on all frontier elements:

1. A $q$-order polynomial representation of the conservative variables, $\overline{\mathbf{U}}(\xi)$, is constructed in the element using Eq. (47).

2. A $(q+1)$-order polynomial representation of the flux, $\overline{\mathbf{E}}(\xi)$, is constructed in the element using Eq. (48).

3. The polynomial is differentiated to provide an estimation of the flux derivative at the boundary's flux points, $\left(\partial_{\xi} \widehat{\mathbf{E}}\right)_{\mathbf{E P}} \mathbf{P}_{q+2}$.

4. Equations (23) and (24) are used to evaluate $\mathcal{N}$ and $\mathcal{S}$ at the boundary's flux points.

5. A corrected $\mathcal{N}^{*}$ is derived from the NSCBC conditions (see Sec. 3).

6. The corrected flux derivative at the boundary's flux points, $\left(\partial_{\xi} \widehat{\mathbf{E}}\right)_{\mathbf{F P}_{q+2}}^{*}$, is evaluated from Eq. (26). The impact of this correction on the flux derivative at the solution points is calculated using one of the two following methods:

(a) Equation (53) is used to evaluate the corresponding corrected flux at the boundary's flux points referred to as $\widehat{\mathbf{E}}_{q+2}^{*}$. Then, the corrected Lagrange polynomial representation of the flux derivative, $\left(\partial_{\xi} \overline{\mathbf{E}}\right)^{*}$ is built using Eq. (49). Finally, $\left(\partial_{\xi} \widehat{\mathbf{E}}\right)_{\mathbf{S P}}^{*}$ is evaluated once again so that the solution, now accounting for the NSCBC, can be marched in time at all the frontier element's solution points.

(b) Alternatively, if the derivative of the Lagrange polynomial is known, $\widehat{\mathbf{E}}_{q+2}^{*}$ can be evaluated using Eq. (54). A corrective term is then added to the flux derivative at all the solution points using Eq. (55) before the solution is marched in time.

\subsection{NSCBC with the flux reconstruction method}

As explained in Sec. 1, another scheme of interest is the flux reconstruction (FR) method which shares many features with SD. Notably, a strong solution of Eq. (46) is seek by using a polynomial representation of the flux derivatives at solution points distributed across elements. This raises the question of whether NSCBC could be implemented in FR in a similar fashion as in SD. 


\subsubsection{The FR scheme}

First, the FR method is presented using the same formalism and configuration as in Sec. 4.1.1. The starting point of the FR method is very similar to the SD method: each element is transformed into a standardized domain where solution points are distributed following a specific pattern. At the start of a timestep, the discrete solution of conservative variables at the $q+1$ solution points reads as $\mathbf{U}_{l}$, with $l \in[1, q+1]$. As no flux points are allocated, a $(q+1)$-long flux vector is directly computed at the solution points as:

$$
\widehat{\mathbf{E}}_{l}=\widehat{\mathbf{E}}\left(\mathbf{U}_{l}\right), \quad l \in[1, q+1]
$$

A continuous flux $\overline{\mathbf{E}}(\xi)$ is constructed from the Lagrangian polynomial representation based on the solution points:

$$
\overline{\mathbf{E}}(\xi)=\sum_{l=1}^{q+1}\left[\mathbf{E}(l) \mathbf{L}_{l, \mathrm{SP}}(\xi)\right], \quad \xi \in[0,1]
$$

Importantly, this polynomial is only of order $q$ contrary to the SD method. At this point, the flux polynomial representation is globally discontinuous, i.e. $\overline{\mathbf{E}}(1)_{[\text {cell } i-1]} \neq \overline{\mathbf{E}}(0)_{[\text {cell } i]}$. Common fluxes are found by resolving Riemann problems at the element $\xi=0$ and $\xi=1$ boundaries. These are referred as $\breve{\mathbf{E}}(0)$ and $\breve{\mathbf{E}}(1)$, respectively, and are different than the extrapolated value $\overline{\mathbf{E}}(0)$ and $\overline{\mathbf{E}}(1)$. Thus, in order to satisfies flux continuity, the polynomial solution $\overline{\mathbf{E}}(\xi)$ is modified by adding corrective terms at the right $(\xi=1)$ and left $(\xi=0)$ element boundaries. These corrections take the form of $(q+1)$-order polynomial which are equal to the correction at their boundary and equal zero at the opposite one. Note that the nature of these corrective polynomial is unspecified, which implies that there exists an infinite number of FR sub-schemes. The discussion on the benefit and drawbacks of using particular classes of polynomial is beyond the scope of this paper, the reader is referred to the aforementioned studies. In the current study, the right boundary (respectively left boundary) corrective polynomial is simply called $\mathbf{C}_{\text {right }}(\xi)$ (respectively $\left.\mathbf{C}_{\text {left }}(\xi)\right)$ and indiscriminately refer to any $(q+1)$-order polynomial verifying:

$$
\left\{\begin{array}{l}
\mathbf{C}_{\text {right }}(0)=0, \\
\mathbf{C}_{\text {right }}(1)=[\breve{\mathbf{E}}(1)-\overline{\mathbf{E}}(1)] \\
\mathbf{C}_{\text {left }}(1)=0, \\
\mathbf{C}_{\text {left }}(0)=[\breve{\mathbf{E}}(0)-\overline{\mathbf{E}}(0)]
\end{array}\right.
$$

Finally, a $(q+1)$-order polynomial reconstruction of the corrected flux is built across the element, $\overline{\overline{\mathbf{E}}}$ :

$$
\overline{\overline{\mathbf{E}}}(\xi)=\overline{\mathbf{E}}(\xi)+\mathbf{C}_{\text {left }}(\xi)+\mathbf{C}_{\text {right }}(\xi)
$$

which can be differentiated at the solution points in order to march the solution in time just as in the SD method.

To conclude, SD and FR methods appear very similar. Notably, the final steps consists in obtaining a $(q+1)$-order polynomial representation of the flux across the element and differentiate it at the solution points. This suggests that NSCBC could be constructed for FR by simply adjusting the algorithm presented in Sec. 4.1.2.

\subsubsection{Semi-discrete algorithm of NSCBC for FR}

As in Sec. 4.1.2, the NSCBC is assumed to be imposed at the frontier element's right boundary $(\xi=1)$. Since there are no flux points in FR, the corrected flux derivative calculated from Eq. (26) at the NSCBC face are simply referred to as $\left(\partial_{\xi} \widehat{\mathbf{E}}\right)_{\text {right }}^{*}$.

In SD, NSCBC are implemented by 1) correcting the flux derivative at the flux points located on the NSCBC face, then 2) evaluating the corresponding corrected flux and 3) communicating this correction to all the solution points in the corresponding frontier element. An algorithm similar to the one presented as method 6.(b) can be implemented in FR. Indeed, there already exists a normalized flux-correction polynomial at the element's boundaries. For the right boundary, it is called $\mathbf{C}_{\text {right }}(\xi)$, and its derivative is known. This polynomial is included within $\overline{\overline{\mathbf{E}}}(\xi)$ which is differentiated and evaluated at the solution points to march the solution in time. Since the NSCBC provides a 
subsequent correction on the $(\xi=1)$-flux derivative, it is suggested that the differentiated right-correction polynom, $\partial_{\xi} \mathbf{C}_{\text {right }}(\xi)$, can be used to directly evaluate a corrected $\left(\partial_{\xi} \widehat{\mathbf{E}}\right)^{*}$ at the solution points. This reads as:

$$
\left(\frac{\partial \widehat{\mathbf{E}}}{\partial \xi}\right)_{\mathbf{S P}}^{*}=\left[\frac{\partial \overline{\overline{\mathbf{E}}}}{\partial \xi}+\frac{\partial \mathbf{C}_{\text {right }}}{\partial \xi} \times\left(\frac{\left(\partial_{\xi} \widehat{\mathbf{E}}\right)_{\text {right }}^{*}-\partial_{\xi} \overline{\overline{\mathbf{E}}}(1)}{\partial_{\xi} \mathbf{C}_{\text {right }}(1)}\right)\right](\mathbf{S P})
$$

This expression satisfies the strong correction on the flux derivative at $\xi=1$, and is analogous to Eq. (55). Importantly, it remains coherent with the essence of the FR scheme as the user-specified polynomial $\mathbf{C}_{\text {right }}$ is, once again, solely bearing the said correction at the frontier element's boundary.

\section{Test cases}

The efficiency of the implementation of NSCBC for the SD method is now assessed by running a series of canonical configurations. In these tests, an incident perturbation of the thermodynamic field will interact with the boundary. It matters not what physical mechanism might have generated such instability (combustion, turbulence, etc), but rather how the NSCBC is able to process it and output the desired reflected signal.

The code considered to perform these tests has undergone extensive bench-marking tests validating its basics functions which can be found in [61, 62]. The solver explicitely marches the solution in time with a 6-stage Runge-Kutta scheme and resolves the Riemann problem at the elements interfaces with a Roe scheme as in [35]. The validation test cases presented herein are designed to use all the NSCBC sub-classes presented in Sec. 3. Namely, the nonreflecting inflow, outflow, isothermall no-slip wall and time-domain impedance characteristic boundary conditions are studied in canonical configurations. Their implementation in the code strictly follows the method presented in Sec. 4.1.2. Further, the auxiliary variables $(\widetilde{\phi}, \widetilde{\psi})$ are allocated in $q$-contiguous arrays as explained in Sec. 3.2 .2 with HPC-applications in mind. Continuous efforts are being made in regard to the code's computational performance and scaling. The use of clever CPU-partitioning can, for instance, designate particular CPUs to resolve heavy-computing NSCBC-neighboring elements.

\subsection{Non-reflecting pressure outflow}

\subsubsection{One-dimensional acoustic wave}

First and foremost, the efficiency of a perfectly non-reflecting boundary condition as given by Eqs. (31) with $K_{p}=0$ is evaluated. The most optimal configuration possible is first investigated. It corresponds to a 1D acoustic wave in a viscous-less quiescent flow at standard temperature and pressure, i.e. $T=300 \mathrm{~K}$ and $p_{0}=1 \mathrm{MPa}$. The wave consists of a $1 \mathrm{~Pa}$-amplitude right-running sine wave of frequency $f=1000 \mathrm{~Hz}$ and wavelength $\lambda=0.347 \mathrm{~m}$. It is initially centered on a $(\mathrm{L}=3 \lambda)$-long domain discretized with 120 elements (i.e. $40 \mathrm{EPW})$. The non-reflecting outlet conditions are applied at $x=0$ and $x=L$.

Figure 1 presents snapshots of the static pressure difference $p^{\prime}=p-p_{0}$ at different time as the wave exits the domain. As can be seen throughout the run, increasing the numerical scheme's order of accuracy (i.e. the polynomial order $q$ ) as a marginal effect on the outlet's reflectivity. In all cases the wave is effectively absorbed. Higher orders of accuracy tend to result in slightly faster absorption of the incident wave as it crosses the boundary as can be observed from the $t=2.6 \mathrm{~ms}$ snapshot. Figure 2 presents the evolution in time of the acoustic energy ratio $E_{a} / E_{a, 0}$. The acoustic energy $E_{a}$ is defined as the integral over the computational domain of the pressure variance. The reference acoustic energy at $t=0$ is called $E_{a, 0}$. While all cases effectively damp the acoustic energy ratio it appears that higher orders of accuracy do so more rapidly. Once the wave has left the domain, the acoustic energy quickly depletes.

\subsubsection{Cylindrical acoustic wave}

A more complex test case is designed as a cylindrical pressure wave propagating in a 2D space using Euler equations. The case is initialized as a $100 \mathrm{~Pa}$ cylindrical pressure step centered on the domain. Uniformly-structured and unstructured meshes are used in order to evaluate the effectiveness of the generalized formulation presented in Sec. 2.1. Both meshes cover a 10x10 $\mathrm{cm}^{2}$ surface and comprise 100x100 elements. Figure 3 presents snapshots of static pressure perturbation $p^{\prime}[\mathrm{Pa}]$ at different times as the wave exits the domain. The unstructured mesh is shown in the first snapshot. The pressure gradient drives the flow in all directions as the wave propagates which triggers a 

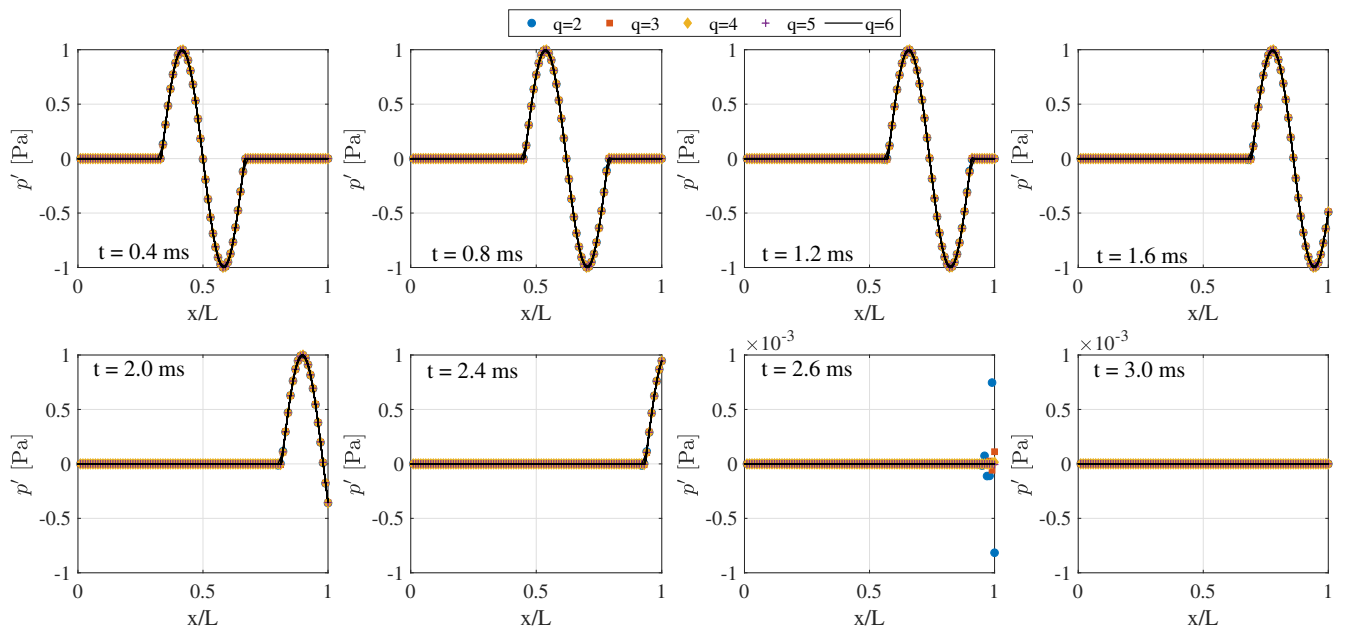

Figure 1: Snapshots of a right-running acoustic wave exiting at $x / L=1$ through a perfectly non-reflecting boundary condition using different polynomial orders $q$.

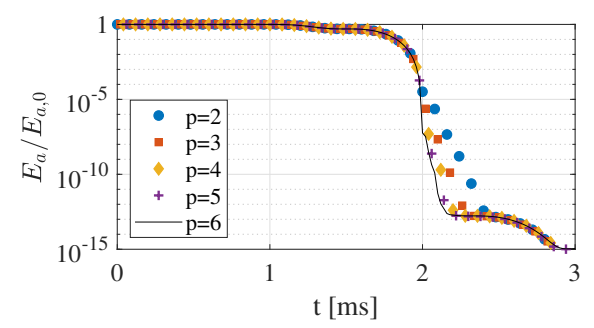

Figure 2: Time-evolution of the acoustic energy ratio inside the computational domain using different polynomial orders $q$.

tangent component at every boundaries. Non-reflecting boundary condition are used on all sides, but only the $x=10$ $\mathrm{cm}$ and $y=10 \mathrm{~cm}$ faces account for the tangent flux contributions as in Eq. (31). That is, the bottom left corner takes no account of the transverse components on either face: it is referred to as the N-N corner. Similarly, the top left and top right corners are referred to as the N-T and T-T corners and respectively account on one and two sides for the transverse components. Each isoline shown in Fig. 3 corresponds to a $2 \mathrm{~Pa}$ level. The contours qualitatively reveal how the $(x, y)=(10,10)$ corner $(\mathrm{T}-\mathrm{T})$ is able to better absorb the cylindrical wave compared to the $(x, y)=(0,0)$ corner $(\mathrm{N}-\mathrm{N})$. The top left and bottom right corners $(\mathrm{N}-\mathrm{T})$ present both a reflectivity in between the N-N and T-T corners and are symmetrical.

Figure 4 presents the evolution in time of the acoustic energy ratio integrated over each quadrant. It shows that accounting for the tangent flux decreases the pressure variance after the wave's passage by an order of magnitude. Importantly, using either a uniform or unstructured mesh does not affect the NSCBC's reflectivity. As mentioned in Sec. 2.1, discarding the mesh corrective terms while using the unstructured mesh has the effect of adding a source terms to the flux derivative at the boundaries, which, when tested, caused the solution to diverge after a few timesteps. This validates the derivation of the NSCBC in generalized coordinates.

\subsection{3. $2 D$ vortex convection}

A testcase often encountered in NSCBC studies [50, 17, 46, 51, 49, 47] is that of an isentropic convecting vortex passing through a non-reflecting pressure outflow. It is now simulated using the Euler equations over a 10x10 cm 2D space centered on $(\mathrm{x}, \mathrm{y})=(0,2.5) \mathrm{cm}$. The bulk flow has a Mach number $M_{\infty}=0.3\left(u_{\infty}=104 \mathrm{~m} / \mathrm{s}\right)$ and is initialized at standard temperature and pressure conditions. A vortex of circulation $\Gamma=34.7 \mathrm{~m}^{2} . \mathrm{s}^{-1}$ and radius $5 \mathrm{~mm}$ at the center of the domain. The initial peak departure velocity $u_{0}=U-u_{\infty}$ equals $0.25 u_{\infty}$ which corresponds to a maximum 

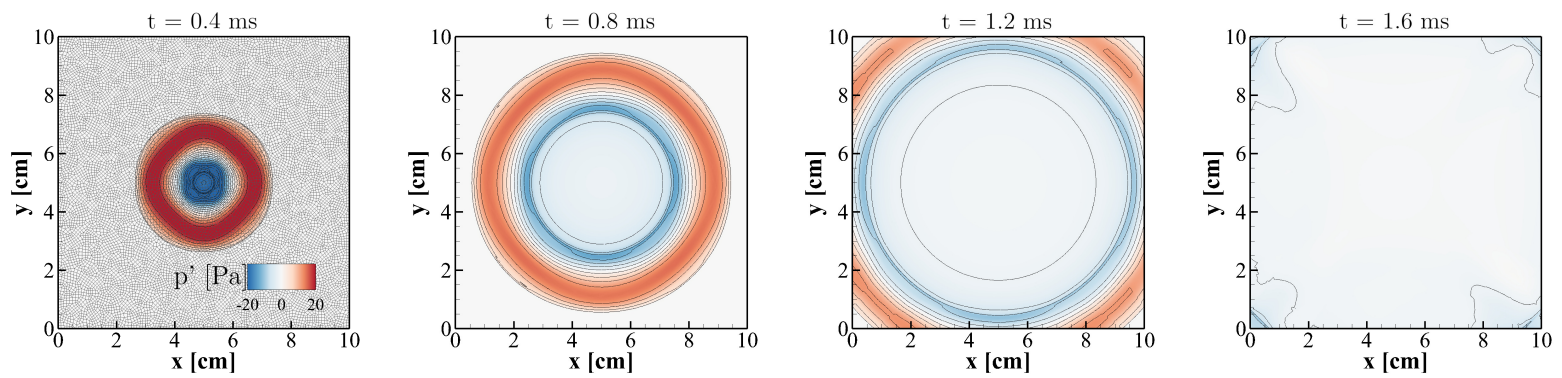

Figure 3: Static pressure contours in time ordered from left to right for a circular pressure wave of initial amplitude 100 Pa. The boundaries are treated as non-reflecting outlets with the $y=10 \mathrm{~cm}$ (top) and $x=10 \mathrm{~cm}$ (right) boundaries only accounting for the tangent fluxes.

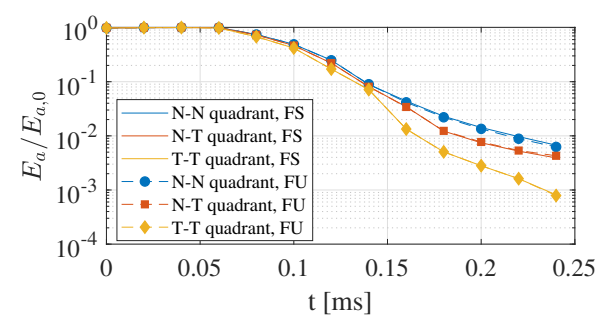

Figure 4: Time-evolution of the acoustic energy inside three quadrants of the computational domain.

pressure defect $p_{0}=698 \mathrm{~Pa}$. In such configuration, the tangent flux is an important component at the boundary. Non-reflecting boundary conditions are imposed at the $x=5 \mathrm{~cm}$ boundary using Eq. (31) with $K_{p}=0$. Conveniently, the configuration yields a bulk exact tangent component $\mathcal{S}_{-}^{\text {exact }}=0$. Determining the optimal transverse contribution factor $\alpha$ used in Eq. (30) is still the focus of ongoing research, and $\alpha=M_{\infty}$ is chosen for the remainder of the study as suggested by numerous studies $[48,47,49,50]$. The $x=-5 \mathrm{~cm}$ side is treated as a perfectly non-reflecting inflow boundary condition (Eqs. (32)) while the $y=-2.5,+7.5 \mathrm{~cm}$ are treated as a non-reflecting outflow with $\alpha=0$. Again, the effect of polynomial order $q$ from 2 to 6 on the reflection amplitude is investigated and the same structured and unstructured meshes from Sec. 5.1.2 are used.

The result of this test case for $q=5$ is presented in Figs. 5 and 6 for the structured and unstructured meshes, respectively. Both meshes are displayed on the top contours which represent snapshots of the normalized y-velocity $v^{*}=v / u_{0}$ as the vortex convects out of the computational domain. The bottom contours represent the normalized pressure departure $p^{*}=\left(p-p_{\infty}\right) / p_{0}$. In these contours, all isolines represent a $1 \%$ level and the minimum and maximum values of the normalized variables are referenced. At $t=0.2 \mathrm{~ms}$, the vortex approaches the boundary. It has begun interacting with it by $t=0.4 \mathrm{~ms}$ which results in a weak spurious distortion of the pressure field for the unstructured case. No such defect is observed in the structured case. At $t=0.6 \mathrm{~ms}$, the reflected acoustic wave is seen propagating backward towards the domain's inflow. With both meshes, the leading reflected wave consists of a over-pressure front caused by the NSCBC's imperfections inducing a numerical stiffness. In the structured case, the reflected wave is strikingly symmetrical around the $\mathrm{y}=0 \mathrm{~cm}$ centerline. At $t=0.8 \mathrm{~ms}$, the reflected wave has exited through the inflow and the computational domain has returned to a quiescent state. The overall result is satisfactory as not only is the reflection amplitude on the order of a few percents but the unstructured mesh behaves almost as well as the uniform one.

The same configuration was then run on set of coarser structured and unstructured meshes both comprising 20x20 elements. First, the snapshots of the coarse structured run are presented in Figs. 7 for $q=5$. It appears that the change of resolution has no noticeable effect on the uniform case. The same symmetrical reflected wave contours are observed with, strikingly, the same $p^{*}$ and $v^{*}$ bounds. Figure 8 present the centerline profiles of the normalized pressure departure $p^{*}$ and vertical velocity $v^{*}$ for several numerical scheme's order of accuracy. Importantly, varying the order of accuracy with the coarse mesh has no noticeable impact on the outlet's reflectivity. The red dotted line correspond to the most accurate case ran $(q=6$ on the fine unstructured mesh) and is shown for reference. Notably, 

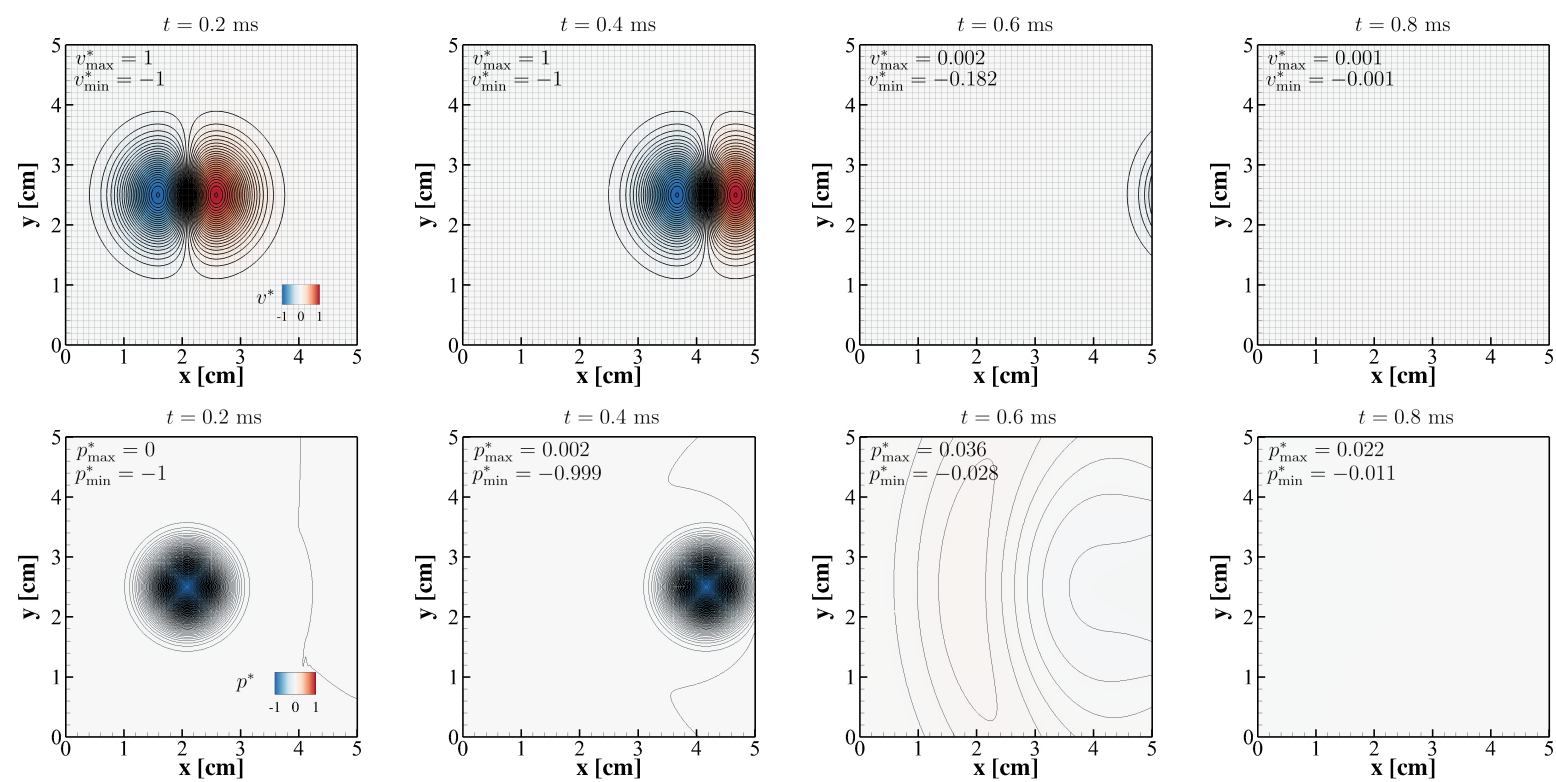

Figure 5: Snapshots of normalized (top) vertical velocity $v^{*}$ and (bottom) departure pressure $p^{*}$ for a vortex convecting through a non-reflecting outlet located at $x=5 \mathrm{~cm}$ on the fine structured mesh.
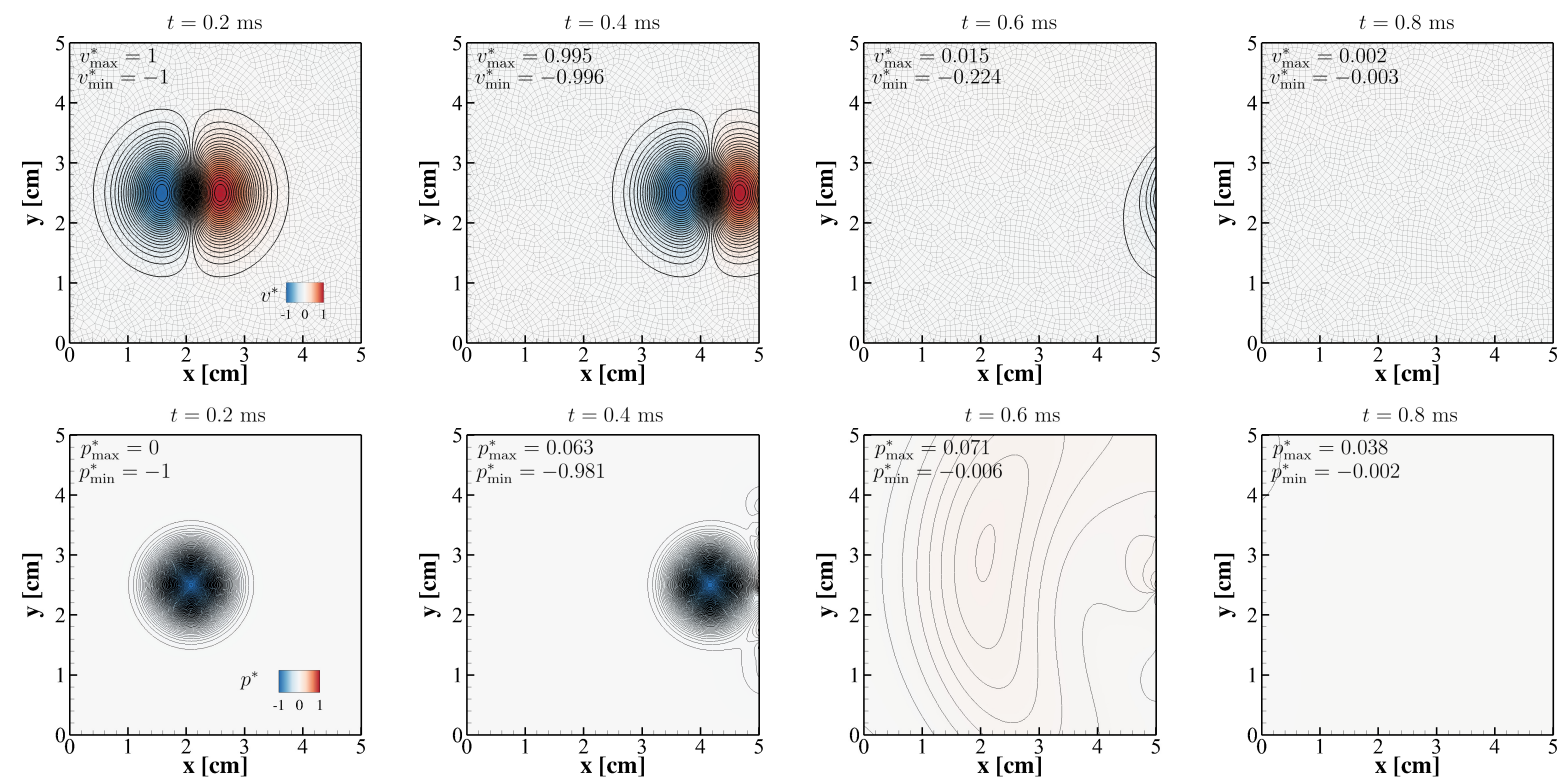

Figure 6: Snapshots of normalized (top) vertical velocity $v^{*}$ and (bottom) pressure departure $p^{*}$ for a vortex convecting through a no-reflecting outlet located at $x=5 \mathrm{~cm}$ on the fine unstructured mesh.

while the lowest accurate case ( $q=2$ on the coarse mesh) presents a strongly distorted vortex, the reflected pressure profile collapse on the reference solution. The effect of the polynomial order is, however, more pronounced on the centerline velocity profiles for $q=2$ which is likely due to the vortex structure having almost broken down at such low resolution. Logically, varying the order of accuracy on the fine unstructured mesh yielded little difference given the spatial resolution was already sufficient. Interestingly, Toulopoulos and Ekaterinaris [15] ran a similar vortex configuration with their implementation of NSCBC with the discontinuous Galerkin method. They observed that 
increasing the order of accuracy increased the boundary's reflectivity as the mesh resolution coarsened.
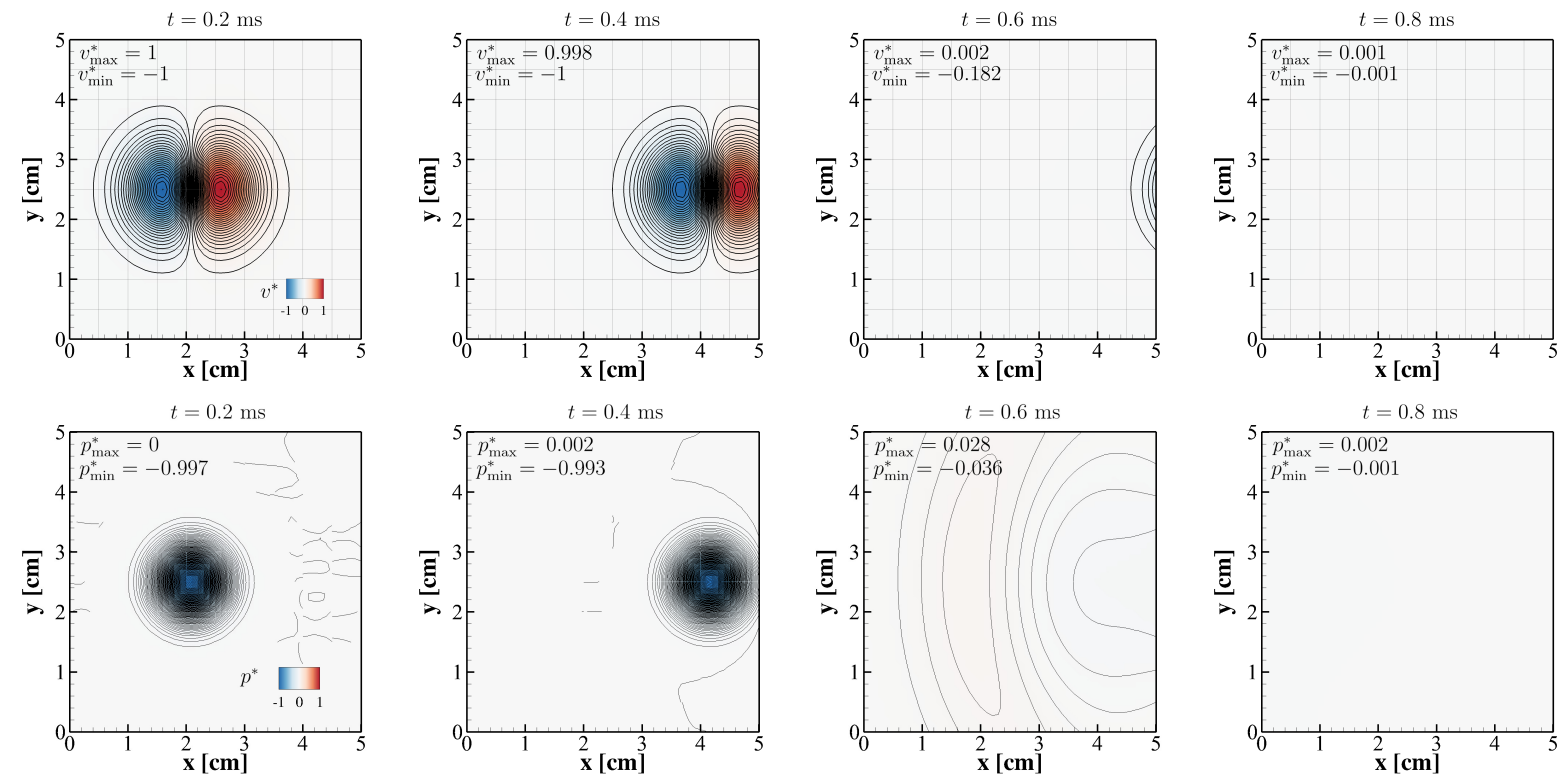

Figure 7: Snapshots of normalized (top) vertical velocity $v^{*}$ and (bottom) pressure departure $p^{*}$ for a vortex convecting through a non-reflecting outlet located at $x=5 \mathrm{~cm}$ on the coarse structured mesh.
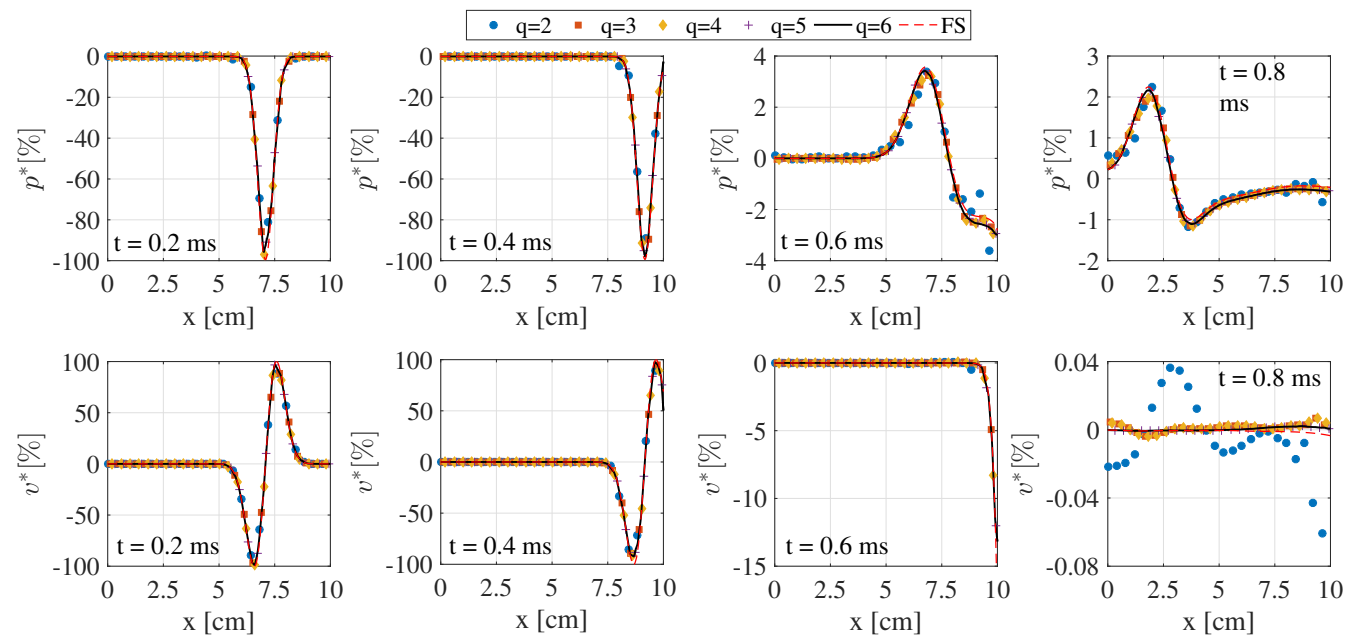

Figure 8: Effect of polynomial order $q$ on the (top) pressure departure $p^{\prime}$ and (bottom) vertical velocity $v$ profiles through time extracted along the $y=2.5 \mathrm{~cm}$ centerline for the coarse uniform mesh. The fine structured $q=6$ solution is presented in red for reference.

The snapshots for the coarse unstructured mesh are presented in Fig. 9 for $q=5$. Contrary to all the other runs, the coarse case now presents a large pressure drift as well as considerable spurious reflections.

It is reminded that, up to this point, perfectly non-reflecting boundary conditions were used (i.e. $K_{p}=0$ ). Both coarse and fine unstructured meshes were then coincidentally used with a positive relaxation rate $K_{p}=0.1,30,75,150,310,780,1500$ resulting in a partially non-reflecting characteristic boundary conditions. This was made in an effort to eliminate the unphysical artifacts observed in Fig. 9. For reference, $K_{p}=780$ corresponds to the value using the expression from Rudy and Strikwerda [63]. The effects of the relaxation rate are illustrated in Fig. 10 which presents snapshots of normalized pressure departure $p^{*}$ at $t=0.6 \mathrm{~ms}$ for different values of $K_{p}$. First, increasing the $K_{p}$ to 30 has roughly 

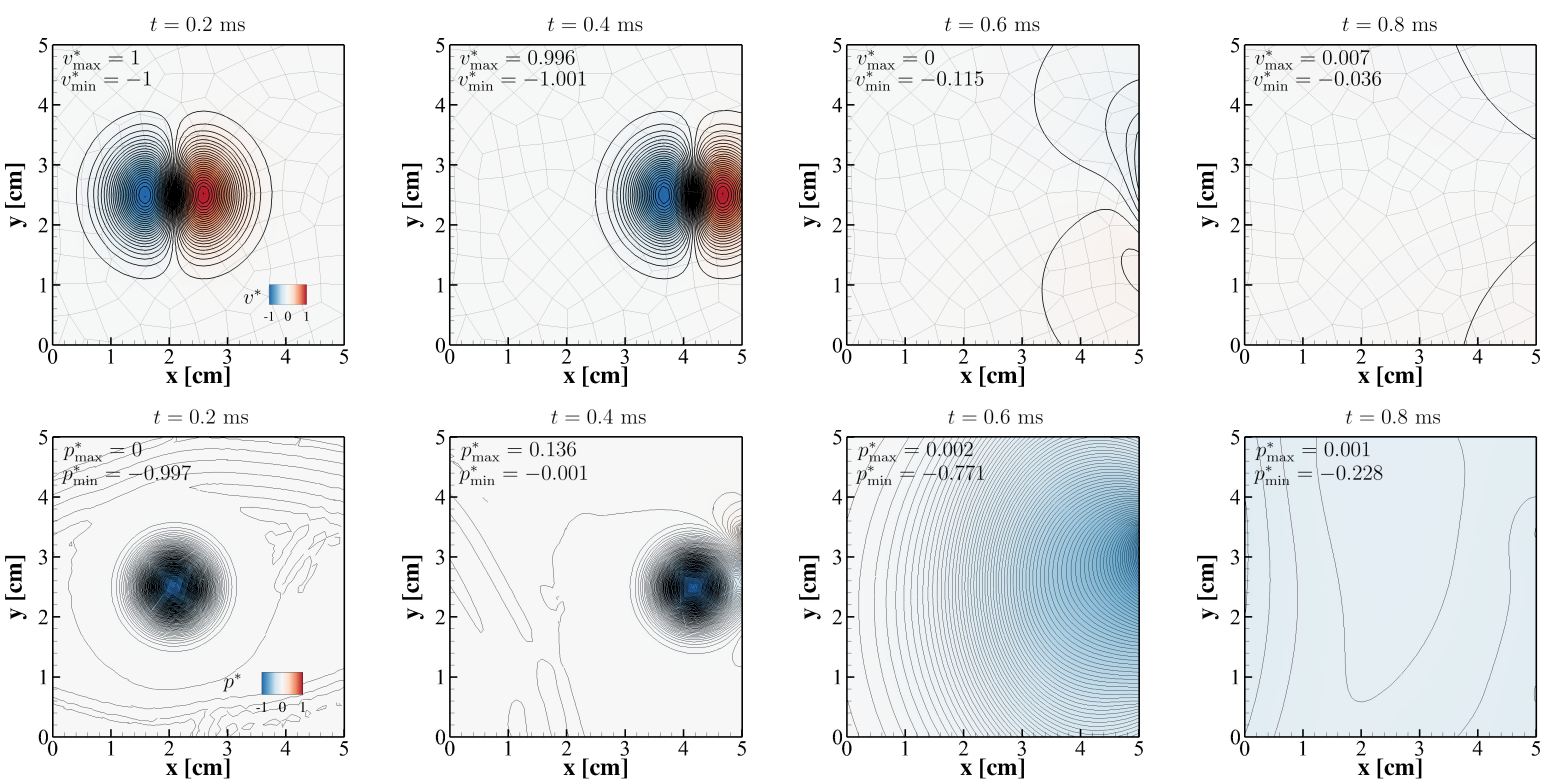

Figure 9: Snapshots of normalized (top) vertical velocity $v^{*}$ and (bottom) pressure departure $p^{*}$ for a vortex convecting through a non-reflecting outlet located at $x=5 \mathrm{~cm}$ on the coarse unstructured mesh for $q=5$.

halved the amplitude of the reflected wave compared to the perfectly non-reflecting case. Yet, the pressure relaxation rate is not strong enough to prevent a large pressure-defect shift after the wave's crossing through the partially nonreflecting outlet. A $K_{p}$ of 150 has significantly reduced both artifacts and the isocontour solution appears to become symmetrical and similar to the coarse structured solution. A $K_{p}$ of 780 results in a quasi-symmetrical solution but has also doubled the reflection amplitude compared to the $K_{p}=150$ case. Further increasing the relaxation rate does not significantly alter the solution which suggest that a convergence has been reached. The said convergence minimizes the pressure drift at the expense of maximizing the reflection wave's amplitude. This implies that an optimal $K_{p}$ coefficient exists and depends on the meshing scheme. It cannot be simply based on the flow configuration as is assumed in [63].
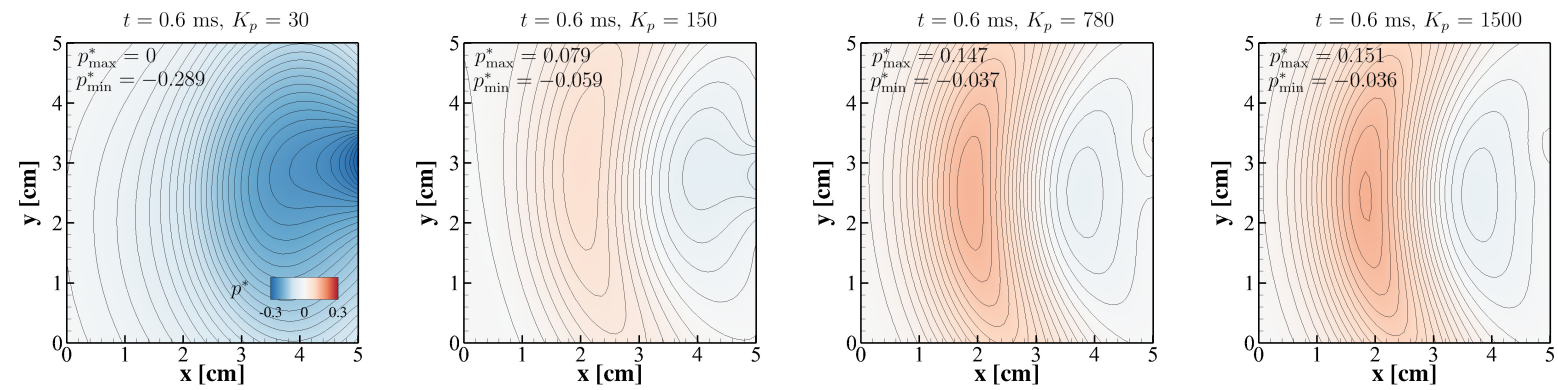

Figure 10: Snapshots of normalized pressure departure $p^{*}$ at $t=0.6 \mathrm{~ms}$ for different relaxation rate $K_{p}$.

The effect of the pressure relaxation rate $K_{p}$ on the outlet's reflectivity was investigated on both the coarse and fine unstructured meshes. The reflectivity is quantified both by the amplitude of the reflected pressure wave calculated as $p_{\max }^{*}-p_{\min }^{*}$ (simply referred to as $\left.\Delta p^{*}\right)$ and the pressure drift calculated as $p_{\max }^{*}+p_{\min }^{*}$ (simply referred to as $\Sigma p^{*}$ ). Note that $p_{\min }^{*} \leq 0$ and $p_{\max }^{*} \geq 0$ was always verified. The results of this sensitivity study are presented in Figs. 11 and 12. Figure 11 shows how these quantities evolves as the vortex convects out of the computational domain, for all the different $K_{p}$ and meshes. Generally, increasing the relaxation rates increases the stiffness on the entering characteristic $W_{-}$. For the fine unstructured mesh, this causes a stronger final $\Delta p^{*}$ and a noticeable $\Sigma p^{*}$ overshoot around $t=0.5 \mathrm{~ms}$. 
For the coarse unstructured mesh, increasing $K_{p}$ progressively decreases the reflection amplitude as the vortex crosses the outflow but increases the final $\Delta p^{*}$. Conversely, it strongly damps the pressure drift $\Sigma p^{*}$. The final $\left(\Delta p^{*}, \Sigma p^{*}\right)$ for the coarse and fine meshes are plotted as a function of $K_{p}$ in Fig. 12. The fine-mesh plots illustrate the well-known compromise one has to consider when determining $K_{p}$. Generally, increasing $K_{p}$ decreases $\Sigma p^{*}$ at the expense of $\Delta p^{*}$. Conversely, the coarse-mesh plots seem to suggest that an optimal $K_{p}$ exists around the value of 75 which effectively limits both pressure reflection and drift amplitudes. Indeed, the coarse mesh with $K_{p}=75$ results in less reflections compared to the finer one using $K_{p}=0$. To conclude, while the determination of an optimal pressure relaxation rate as a function of the mesh is not fully understood yet, partially non-reflecting boundary conditions retain their robustness at very coarse spatial resolutions with either uniform or unstructured meshes.
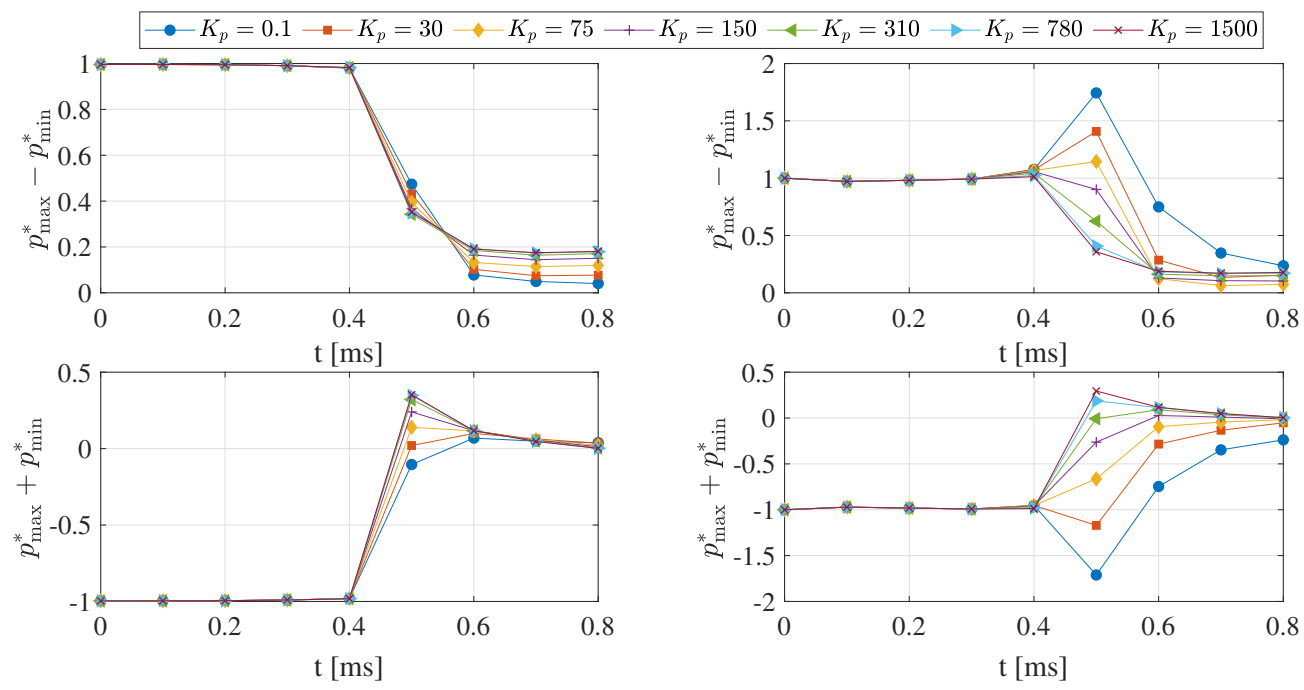

Figure 11: Effect of $K_{p}$ on the time-evolution of the (top) reflection amplitude and (bottom) pressure drift for the (left) fine and (right) coarse unstructured meshes.

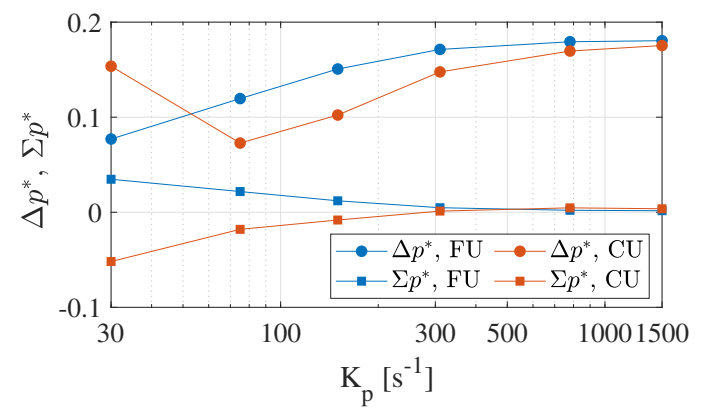

Figure 12: Effect of $K_{p}$ on the final reflection amplitudes and pressure drifts for the (blue) fine and (red) coarse unstructured meshes.

Finally, as the 2D convecting vortex configuration is a popular bench-mark test to study NSCBC, the current implementation is compared with that of Fosso et al. [50] and Granet et al. [49]. All codes have implemented Eqs. (30) with $\alpha=M_{\infty}$ and the same meshes as described in [50,49] are used. However, $K_{p}=0$ is retained in the current implementation contrary to these studies which use the expression from Rudy and Strikwerda [63]. This should provide the lowest $\Delta p^{*}$ and highest $\Sigma p^{*}$ limiting solution. The results are summarized in Tab. 1 where $u_{0} / u_{\infty}$ is the vortex strength relative to the bulk flow. All the quantities are extracted at the same physical times as in their respective papers. The current implementation for strong discontinuous spectral methods appears to be at least as effective as those from finite volume solvers. 


\begin{tabular}{lllllll}
\hline Case & $M_{\infty}$ & $u_{0} / u_{\infty}$ & $p_{\max }^{*}$ & $p_{\min }^{*}$ & $\Delta p^{*}$ & $\Sigma p^{*}$ \\
\hline Granet et al. [49] & 0.028 & $50 \%$ & $0.09[0.01]$ & $-0.08[-0.01]$ & $0.17[0.01]$ & $0.01[0.00]$ \\
Granet et al. [49] & 0.28 & $50 \%$ & $0.09[0.03]$ & $-0.05[-0.03]$ & $0.14[0.06]$ & $0.04[0.00]$ \\
Fosso et al. [50] & 0.5 & $75 \%$ & $0.05[0.04]$ & $-0.03[-0.02]$ & $0.08[0.06]$ & $0.02[0.02]$
\end{tabular}

Table 1: Quantification of NSCBC reflectivities for a 2D convecting vortex from various configurations and studies. The values between brackets correspond to those obtained from the method presented in this study.

\subsection{Impedance wall characteristic boundary conditions}

\subsubsection{No-slip hard viscous wall}

First and foremost, the common no-slip hard wall boundary condition is tested. It amounts to a fully-reflective boundary condition: the entering (i.e. reflected) acoustic characteristic is set to equal the exiting (i.e. incident acoustic characteristic) [64]. Hence, it is a particular case of the TDIBC with $\mathcal{B}=\mathbb{1}$ (or infinite reactance). This condition reads as:

$$
\mathcal{N}_{-}^{*}=\mathcal{N}_{+}-\left(\mathcal{S}_{-}-\mathcal{S}_{+}\right)
$$

A 2D flat plate configuration was run with $q=3$ at Mach 0.3 and standard temperature and pressure conditions using NSE to verify that a correct Blasius profile would be recovered from Eq. (61). Inflow conditions were prescribed using Eqs. (32) and non-reflecting boundary were used at the outflow boundaries. The wall was treated as a $300 \mathrm{~K}$ isothermal no-slip TDIBC with $\mathcal{B}=\mathbb{1}$. Finally, the computational domain is $2 \mathrm{~cm}$ long along the streamwise direction, including $1.6 \mathrm{~cm}$ of wall as the first $0.4 \mathrm{~cm}$ are treated with symmetrical boundary conditions.

Once all transient perturbations have left the computational domain and the solution has converged in time, the wall normal velocity is extracted at different streamwise locations. As shown in Fig. 13, all profiles collapse on the Blasius solution. Importantly, a null normal velocity at the wall is correctly recovered without ever being explicitly prescribed which validates the current approach.

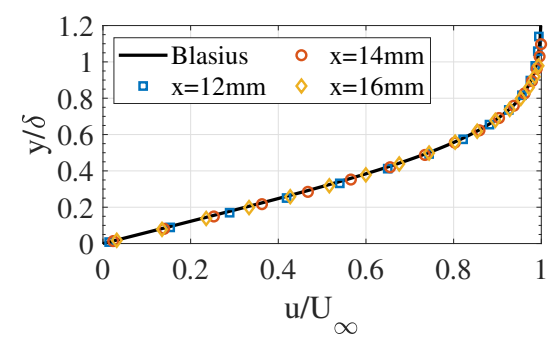

Figure 13: Wall-normal profiles at various streamwise locations $x$ of the normalized streamwise velocity ratio $u / u_{\infty}$ over a hard-wall characteristic boundary condition.

\subsubsection{Permissive viscous boundary condition}

The second wall test case is that of a permissive viscous boundary, i.e. a TDIBC with $\mathcal{B} \in[0,1]$. It is designed to assess the assumption of accounting for the diffusive fluxes within the reflection operator, as done in Eq. (44), where it is reminded that $\mathcal{N}=\mathcal{L}+\mathcal{D}$. Indeed, it could intuitively be argued that this operator, acoustic by nature, should only apply to the convective terms which determine wave propagation phenomenon. This amounts to decouple the diffusive flux with the TDIBC and treat them an an external source terms in the characteristic form of the NavierStokes equations, as is often done [46, 47]. To illustrate the problem this assumption causes, let us consider the typical $(\mathcal{L}, \mathcal{D})$ characteristic determinations as in Eq. (9) in a one-dimensional case. Decoupling (respectively coupling) the diffusive flux with the TDIBC results in a condition on the reflected $\mathcal{L}_{-}^{*}\left(\right.$ respectively $\left.\left(\mathcal{L}_{-}+\mathcal{D}_{-}\right)^{*}\right)$ referred to as the split (respectively compact) formulation. They both read in 1D as: 


$$
\begin{cases}\mathcal{L}_{-}^{*}=\mathcal{B}\left[\mathcal{L}_{+}\right]-\left(\mathcal{D}_{-}-\mathcal{D}_{+}\right), & (\text {split formulation) } \\ \left(\mathcal{L}_{-}+\mathcal{D}_{-}\right)^{*}=\mathcal{B}\left[\mathcal{L}_{+}+\mathcal{D}_{+}\right], & \text {(compact formulation) }\end{cases}
$$

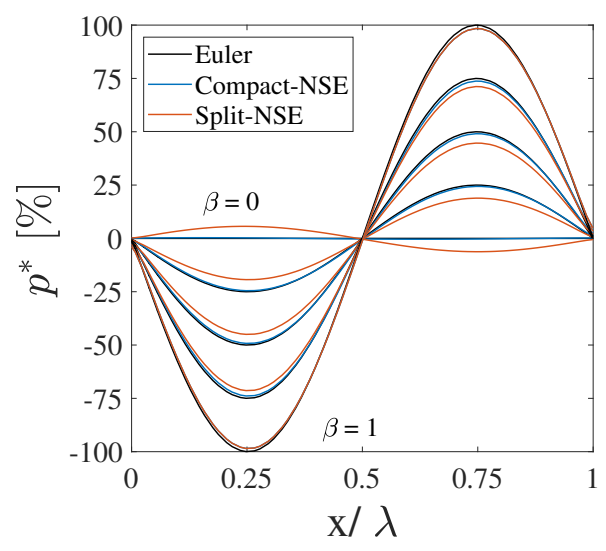

Figure 14: Profiles of reflected acoustic pressure waves of initial amplitude $100 \mathrm{~Pa}$ on a TDIBC defined by $\beta \in[0,0.25,0.5,0.75,1]$ using Euler and the compact or split formulation of the characteristic Navier-Stokes equations.

A $1 \mathrm{D}$ impedance tube configuration (i.e. no tangent fluxes) is now simulated. A 1 Pa right-running sine wave of frequency $f \in[1000] \mathrm{Hz}$ is initialized at the center of a $1 \mathrm{D}$ mesh, yielding a maximum velocity $u_{0}=0.0025$ $\mathrm{m} . \mathrm{s}^{-1}$. The computational domain spans 3 wavelengths and is discretized with 40 EPW. The $x=0$ boundary is treated as a perfectly non-reflecting pressure outlet and the $x=L$ boundary is the TDIBC. Several TDIBC are used, all determined by a real reflection coefficient $\beta \in[0,0.25,0.5,0.75,1]$. In this particular case, both $\beta$ and $\mathcal{B}$ operators are equivalent. Both split and compact Navier-Stokes formulations are run alongside an Euler reference solution. The viscosity, evaluated using Sutherland's law, is multiplied by a factor of ten to emphasize the differences between the formulations and a $6^{\text {th }}$-order SD scheme $(q=5)$ is used.

The right-running wave hits the TDIBC and becomes a left-running reflected pressure wave. The latter is sampled in space once it has complete the back-and-forth travel and returned to its initial position. The resulting reflected profiles are shown in Fig. 14. The issue with the split formulation appears in the form of a spurious over-pressure reflection. It results from the mathematical imbalance caused by the decoupling of the diffusive/convective fluxes which both determine the strength of the incident/reflected characteristics. Strikingly, when $\beta=0$ (i.e. $\mathcal{B}=0$ ) the Euler and Navier-Stokes compact formulation recover a non-reflecting solution given by Eq. (28) (albeit the isothermal wall condition adds some reflectivity) contrary to the Navier-Stokes split formulation. Further, it is seen that the amplitude of the spurious reflection scales with $\beta$. That is, it is maximum for $\beta=0$ and absent for $\beta=1$. Interestingly, this proves that both formulation are equivalent for $\beta=1(\mathcal{B}=1)$ which corresponds to the limiting case of a fully-reflective boundary condition as previously demonstrated. Finally, a 2 Pa peak was measured over time at the TDIBC for the correct formulation. Its corresponds to the expected wave's pressure-doubling caused by constructing interference between incident and reflected waves. However, a higher value was sampled for the incorrect split formulation which further validates the compact approach for NSCBC-embedded TDIBC schemes.

Importantly, these last two testcases verify the continuity of the $\mathcal{B}$-operator between its physical bounds: both non-reflecting and fully-reflecting characteristic boundary conditions are shown to to be recovered from TDIBC with null/identity $\mathcal{B}$.

\subsubsection{Plane-wave grazing over an acoustic liner}

Finally, a numerical experiment mimicking the configuration of Jones et al. [57] is carried out to validate the use of TDIBC with the Navier-Stokes equations on an unstructured mesh. It simulates the effect that an acoustic liner has on a grazing right-running planar wave. This popular bench-marking case has been used by Richter et. al [65], Zhong et. al [66], Monteghetti et al. [54] and Burak et al. [67] to assess the accuracy of their TDIBC. In the current case, 
we use the same two-poles oscillo-diffusive representation as used in [54], that is the same $\left(\beta_{\infty}, s, \xi, r, \mu, \tau\right)$ set which defines the TDIBC, and resolve the delay with a $6^{\text {th }}$-order SD scheme $(q=5)$ and $16 \mathrm{EPW}$.

The configuration of the numerical experiment is presented in Fig. 15. The domain uses non-reflective boundary conditions at the inlet and outlet, fully-reflective hard wall conditions at the walls and a partially-reflective TDIBC to represent the liner's porous surface. All these boundary conditions are implemented as NSCBC, and both NavierStokes (no-slip walls) and Euler (slip walls) equations are considered. The simulation uses a $5^{\text {th }}$-order SD scheme $(q=4)$. Finally, both uniform and unstructured meshes are used to demonstrate the TDIBC capability in working as designed in generalized coordinates.

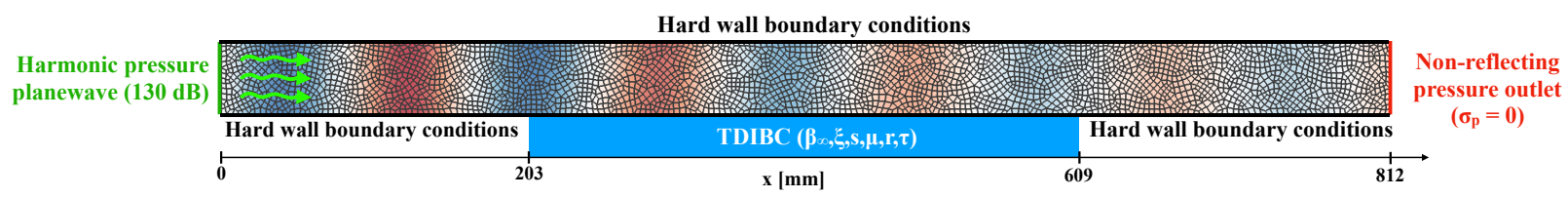

Figure 15: Configuration of the two-dimensional acoustic liner mimicking the experiment of Jones et al. [57]. A snapshot of the normalized static pressure perturbation $p^{*}$ from -1 (blue) to +1 (red) for the $2000 \mathrm{~Hz}$ case are shown with the unstructured mesh.

The simulations run as follows. A train of harmonic right-running acoustic waves of amplitude $130 \mathrm{~dB}$ enters the domain at $t=0$ on the left boundary and exits it on the right. Four different inlet frequencies are investigated: 1000, 1500, 2000 and $3000 \mathrm{~Hz}$. Importantly, the same meshes (unstructured and uniform) are used for all runs resulting in, respectively, an averaged number of $85.5,57.0,42.7$ and 28.5 elements per wavelength. The bulk flow is at rest at atmospheric conditions. The unstructured mesh is displayed in Fig. 15 on top of a static pressure snapshot for the $2000 \mathrm{~Hz}$ case. A sanity check without TDIBC is also run resulting in an unperturbed harmonic train wave exiting the domain with an amplitude of $129.9996 \mathrm{~dB}$. This indicates minimal amount of dissipation and, more importantly, marginal spurious reflections from both open ends of the computational domain.

The liner's absorption efficiency is then evaluated by measuring the sound pressure level on the opposite wall once all transients have been washed out of the computational domain and a permanent regime been established. The best absorption is achieved at $1000 \mathrm{~Hz}$.

These wall profiles are compared in Fig. 16 with the experimental data from [57] and with the linearized Euler solution from [54]. All sound pressure levels closely match with the experimental measurements for all the frequencies considered. Interestingly, the full Navier-Stokes simulations also seem to better capture these profiles than the linearized Euler equation case from [54]. As expected, the $1000 \mathrm{~Hz}$ case achieves the highest absorption with a 62 $\mathrm{dB}$ reduction. This means a reduction in wall pressure perturbation amplitude over three orders of magnitude. Such reduction could only be measured in a sterilized numerical environment void of any spurious noise: this serves as a testament to the value of combining high-order schemes with NSCBC.

Importantly, the aforementioned bench-marking studies ([66, 65, 54]) also correctly recovered the wall sound pressure level profiles, albeit by making the following assumptions:

- The linearized Euler equations were used by $[65,67,66,54]$, as opposed to the Navier-Stokes equations for the present study. Burak et al. [67] attempted to resolve the full Navier-Stokes but could not replicate the flow environment faithfully, and had to resort to use slip conditions on all surfaces. Despite this Eulerian assumption, their LES at $1000 \mathrm{~Hz}$ was not able to measure a wall sound pressure level below $78 \mathrm{~dB}$ while the experiment measured a reduction down to $68 \mathrm{~dB}$. After linearizing the equations they managed to reduce the noise down to $68 \mathrm{~dB}$ as well. This suggests that the nonlinearity of the full Navier-Stokes equation can cause an incompressible level of noise in the simulation which pollutes the acoustic field. Such noise was not noticeable in the other cases where the $\mathrm{dB}$ did not fall below $100 \mathrm{~dB}$. This example perfectly illustrates the need to associate high-order schemes and TDIBC with non-reflection pressure outflow conditions as well.

- An impedance $\mathcal{Z}$-based TDIBC was derived in $[66,65,67]$, which was shown to be more prone to instabilities and CFL restrictions than a reflection $\beta$-based TDIBC [54].

- A three-coefficient model was used by $[66,65,67]$ which required a separate optimization to match the different 

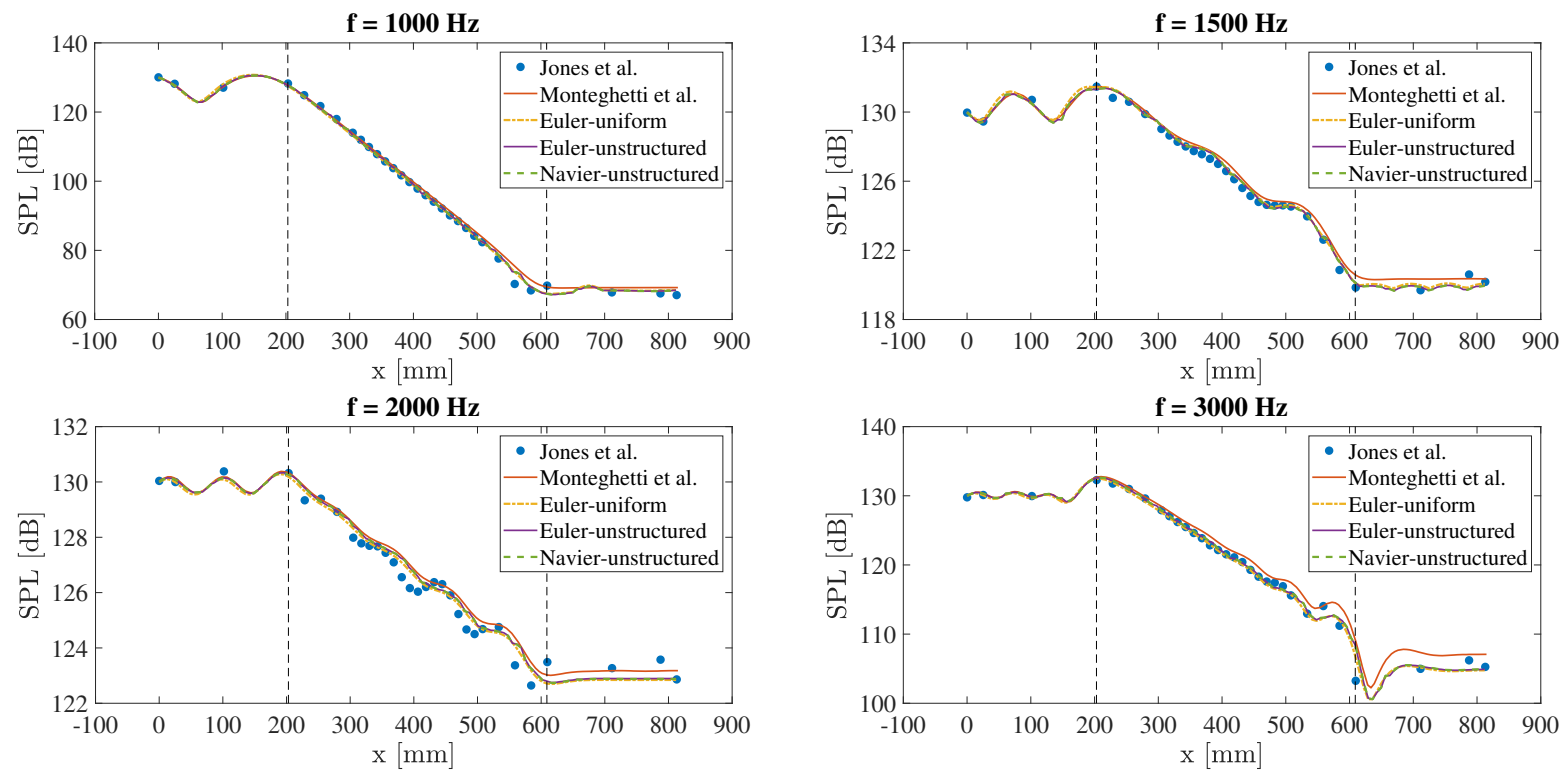

Figure 16: Sound pressure levels measured along the top wall for an incident harmonic wave-train at various frequencies. The acoustic liner's boundaries are marked by the black dashes lines.

cases: on the other hand the multi-pole models require one set of coefficient for the all spectral range of interest and truly qualifies as a broadband model.

To conclude, the current implementation of the TDIBC cumulates the advantages of these aforementioned studies (broadband accurate model based on the $\beta$-formulation coupled with the full Navier-Stokes equations) and more (high-order methods on arbitrarily complex geometries) with none of their short-comings.

\section{Summary and conclusions}

The characteristic boundary conditions for the Navier-Stokes equations [17] have been implemented with strong discontinuous spectral methods, namely the spectral difference and flux reconstruction schemes. These conditions are derived from the divergent form of the NSCBC in generalized coordinates from Kim and Joo [46] and are applied to the flow field as a flux balance correction. In order to be compatible with the numerical schemes, the correction was rewritten in a novel compact polynomial form applied on all the solution points of a frontier element presented in Eqs. (55) and (60) for SD and FR, respectively. The element-compact formulation does not discriminate between convective and diffusive fluxes, which further alleviates the memory and computational costs of the method and simplifies its implementation. Typical bench-marking cases were run in SD to assess the quality of the NSCBC, that is its capacity in enforcing the target reflectivity at a boundary. It performed remarkably well in 1D configurations and performed sensibly better on 2D inhomegeneous flows than similar finite volume studies [49, 50]. Unsurprisingly, the method becomes weakly reflective in the latter case, which is a known limitation inherent to the one-dimensionality of the characteristic decomposition method [17]. Importantly, the quality of the boundary condition was shown to improve as the correction polynomial's order increases.

Time-domain impedance boundary conditions were also developed, embedded within the compact NSCBC module. The discretization of the impedance model in the time-domain is based on the oscillo-diffusive representation from Monteghetti et al. [53] which permits to approximate broadband models of arbitrary complexity into a sum of delayed pole functions. Increasing the number of poles permits to enhance the spectral resolution and resolve abritrarily more complex models, accounting for diffraction and thermo-viscous losses for instance. Importantly, the contribution of each pole to the TDIBC's reflectivity is independently resolved in the time-domain as an auxiliary 
function solution of ODE and PDE which can conveniently use the same time-marching scheme as the main simulation. The main novelty of the TDIBC implementation lies in its semi-discrete coupling with the full Navier-Stokes equations: it addresses the treatment of the diffusive flux at the wall and does not rely on the use of perturbations variables with respect to a mean flow, as is proper to a non-linear time-resolved simulation. As a NSCBC-subclass, it also benefits from an increase of the correction polynomial order.

To conclude, characteristic boundary conditions for Navier-Stokes equations have been successfully used with the spectral difference method for the first time. An implementation scheme with the flux reconstruction method was also suggested and shown to closely follow that for SD. The NSCBC are built from a strong compact polynomial form of the conservation equations, which also serves as a semi-discrete coupling scheme for impedance (i.e. frequencydependent) conditions with the Navier-Stokes equations. The development of such boundary conditions for strong discontinuous spectral methods enables their use in computational aeroacoustics, laminar-turbulence transition and other highly-demanding unsteady problems on complex geometries.

\section{Acknowledgement}

The authors gratefully acknowledge the financial support given by the STAE foundation (Science and Technology for Aeronautics and Space) through grant number CDT-R050-L00-T00. This work is part of the 3C2T project (control of compressible, transitional and turbulent boundary layer) involving the the French aerospace laboratory (ONERA), the European Center for Research and Formation in Advanced Scientific Calculus (CERFACS) and the Institute of Fluid Mechanics of Toulouse (IMFT) under the supervision of Christophe Airiau (IMFT). The spectral difference flow solver used throughout this study is named JAGUAR (proJect of an Aerodynamic solver using General Unstructured grids $A$ nd high orde $R$ schemes) and is actively co-developed by CERFACS and ONERA. The authors thank Michael G. Jones for providing the database of measurements of the NASA grazing incidence tube experiment. Finally, the authors also thank Guillaume Puigt (ONERA) for fruitful discussions on the spectral difference and flux reconstruction methods.

\section{Appendix: Transformation matrices for Navier-Stokes equations}

The transformation from the physical $\mathbf{x}=(x, y, z)$ space into the iso-parameteric $\xi=(\xi, \eta, \zeta)$ space reads as:

$$
\boldsymbol{\xi}_{\mathbf{x}}=\left[\begin{array}{ccc}
\xi_{x} & \xi_{y} & \xi_{z} \\
\eta_{x} & \eta_{y} & \eta_{z} \\
\zeta_{x} & \zeta_{y} & \zeta_{z}
\end{array}\right]=J\left[\begin{array}{lll}
y_{\eta} z_{\zeta}-y_{\zeta} z_{\eta} & z_{\eta} x_{\zeta}-z_{\zeta} x_{\eta} & x_{\eta} y_{\zeta}-x_{\zeta} y_{\eta} \\
y_{\zeta} z_{\xi}-y_{\xi} z_{\zeta} & z_{\zeta} x_{\xi}-z_{\xi} x_{\zeta} & x_{\zeta} y_{\xi}-x_{\xi} y_{\zeta} \\
y_{\xi} z_{\eta}-y_{\eta} z_{\xi} & z_{\xi} x_{\eta}-z_{\eta} x_{\xi} & x_{\xi} y_{\eta}-x_{\eta} y_{\xi}
\end{array}\right]
$$

where the Jacobian $J$ is calculated from the grid metrics as

$$
J=\left[x_{\xi}\left(y_{\eta} z_{\zeta}-y_{\zeta} z_{\eta}\right)+x_{\eta}\left(y_{\zeta} z_{\xi}-y_{\xi} z_{\zeta}\right)+x_{\zeta}\left(y_{\xi} z_{\eta}-y_{\eta} z_{\xi}\right)\right]^{-1}
$$

The transformation matrices used to change between conservative $\mathbf{U}$, primitive $\mathbf{Q}$ and characteristic variables $\mathbf{W}$ are given below for a calorically perfect gas. The method can easily be extended to thermally perfect gas. The inverted matrices are used for the inverse transforms. As the characteristics are expressed in terms of primitive variables $\mathbf{Q}$, it is more intuitive to first convert the Navier-Stokes from conservative to primitive variable space. This transformation matrix, referred to as $\frac{\partial \mathbf{Q}}{\partial \mathbf{U}}$, reads as:

$$
\frac{\partial \mathbf{Q}}{\partial \mathbf{U}}=\left[\begin{array}{ccccc}
1 & 0 & 0 & 0 & 0 \\
-u / \rho & 1 / \rho & 0 & 0 & 0 \\
-v / \rho & 0 & 1 / \rho & 0 & 0 \\
-w / \rho & 0 & 0 & 1 / \rho & 0 \\
\frac{1}{2}|\mathbf{u}|^{2}(\gamma-1) & -(\gamma-1) u & -(\gamma-1) v & -(\gamma-1) w & \gamma-1
\end{array}\right]
$$


The transformation matrix from primitive to characteristic space, $P_{\mathbf{Q}}$, projected on a face with normal $\left(n_{x}, n_{y}, n_{z}\right)$, reads as:

$$
P_{\mathbf{Q}}=\frac{\partial \mathbf{W}}{\partial \mathbf{Q}}=\left[\begin{array}{ccccc}
n_{x} & 0 & n_{z} & -n_{y} & -n_{x} / c^{2} \\
n_{y} & -n_{z} & 0 & n_{x} & -n_{y} / c^{2} \\
n_{z} & n_{y} & -n_{x} & 0 & -n_{z} / c^{2} \\
0 & \rho c n_{x} & \rho c n_{y} & \rho c n_{z} & 1 \\
0 & -\rho c n_{x} & -\rho c n_{y} & -\rho c n_{z} & 1
\end{array}\right]
$$

Finally the transformation matrix $P_{\mathbf{U}}$ which equals $\frac{\partial \mathbf{W}}{\partial \mathbf{U}}$ is given by $P_{\mathbf{U}}=P_{\mathbf{Q}} \times \frac{\partial \mathbf{Q}}{\partial \mathbf{U}}$.

The inverse transformation matrices are given as:

$$
\frac{\partial \mathbf{U}}{\partial \mathbf{Q}}=\left[\begin{array}{ccccc}
1 & 0 & 0 & 0 & 0 \\
u & \rho & 0 & 0 & 0 \\
v & 0 & \rho & 0 & 0 \\
w & 0 & 0 & \rho & 0 \\
\frac{1}{2}|\mathbf{u}|^{2} & \rho u & \rho v & \rho w & 1 /(\gamma-1)
\end{array}\right]
$$

and

$$
\frac{\partial \mathbf{Q}}{\partial \mathbf{W}}=\left[\begin{array}{ccccc}
n_{x} & n_{y} & n_{z} & 1 /\left(2 c^{2}\right) & 1 /\left(2 c^{2}\right) \\
0 & -n_{z} & n_{y} & n_{x} /(2 \rho c) & -n_{x} /(2 \rho c) \\
n_{z} & 0 & -n_{x} & n_{y} /(2 \rho c) & -n_{y} /(2 \rho c) \\
-n_{y} & n_{x} & 0 & n_{z} /(2 \rho c) & -n_{z} /(2 \rho c) \\
0 & 0 & 0 & 1 / 2 & 1 / 2
\end{array}\right]
$$

[1] F. Kerhervé, P. Jordan, A. V. G. Cavalieri, J. Delville, C. Bogey, D. Juvé, Educing the source mechanism associated with downstream radiation in subsonic jets, Journal of Fluid Mechanics 710 (2012) $606-640$.

[2] D. A. Buchta, J. B. Freund, The near-field pressure radiated by planar high-speed free-shear-flow turbulence, Journal of Fluid Mechanics 832 (2017) $383-408$.

[3] C. Bogey, On noise generation in low reynolds number temporal round jets at a mach number of 0.9, Journal of Fluid Mechanics 859 (2019) $1022-1056$.

[4] J. H. Chen, A. Choudhary, B. de Supinski, M. DeVries, E. R. Hawkes, S. Klasky, W. K. Liao, K. L. Ma, J. Mellor-Crummey, N. Podhorszki, R. Sankaran, S. Shende, C. S. Yoo, Terascale direct numerical simulations of turbulent combustion using s3d, Computational Science \& Discovery 2 (2009) $001-015$.

[5] H. Wang, E. R. Hawkes, B. Zhou, J. H. Chen, Z. Li, M. Aldn, A comparison between direct numerical simulation and experiment of the turbulent burning velocity-related statistics in a turbulent methane-air premixed jet flame at high karlovitz number, Proceedings of the Combustion Institute 36 (2017) 2045 - 2053.

[6] T. Poinsot, Prediction and control of combustion instabilities in real engines, Proceedings of the Combustion Institute 36 (2017) $1-28$.

[7] A. D. Beck, T. Bolemann, D. Flad, H. Frank, G. J. Gassner, F. Hindenlang, C.-D. Munz, High-order discontinuous galerkin spectral element methods for transitional and turbulent flow simulations, International Journal for Numerical Methods in Fluids 76 (2014) 522 - 548.

[8] J. Sivasubramanian, H. F. Fasel, Direct numerical simulation of transition in a sharp cone boundary layer at mach 6: fundamental breakdown, Journal of Fluid Mechanics 768 (2015) $175-218$.

[9] A. Frére, N. N. Sørensen, K. Hillewaert, G. Winckelmans, Discontinuous galerkin methodology for large-eddy simulations of wind turbine airfoils, Journal of Physics: Conference Series 753 (2016) 022 - 037

[10] C. Anghan, S. Dave, S. Saincher, J. Banerjee, Direct numerical simulation of transitional and turbulent round jets: Evolution of vortical structures and turbulence budget, Physics of Fluids 31 (2019) $065-105$.

[11] W. Reed, Y. Wang, A. Trouvé, H. G. Im, Triangular mesh methods for the neutron transport equation, in: National topical meeting on mathematical models and computational techniques for analysis of nuclear systems (1973).

[12] B. Cockburn, C.-W. Shu, Runge-kutta discontinuous galerkin methods for convection-dominated problems, Journal of Scientific Computing $16(2001) 173-261$.

[13] Y. Lv, M. Ihme, Discontinuous galerkin method for multicomponent chemically reacting flows and combustion, Journal of Computational Physics 270 (2014) $105-137$.

[14] Y. Lv, M. Ihme, High-order discontinuous Galerkin method for applications to multicomponent and chemically reacting flows, Acta Mechanica Sinica 33 (2017) 486-499.

[15] I. Toulopoulos, J. A. Ekaterinaris, Artificial boundary conditions for the numerical solution of the euler equations by the discontinuous galerkin method, Journal of Computational Physics 230 (2011) 5974 - 5995.

[16] K. W. Thompson, Time dependent boundary conditions for hyperbolic systems, Journal of Computational Physics 68 (1987) $1-24$.

[17] T. J. Poinsot, S. K. Lele, Boundary conditions for direct simulations of compressible viscous flows, Journal of Computational Physics 101 (1992) 104-129.

[18] C. K. W. Tam, L. Auriault, Time-domain impedance boundary conditions for computational aeroacoustics, AIAA Journal 34 (1996) 917 923.

[19] C. Richter, J. Abdel-Hay, Åukasz Panek, N. Schnwald, S. Busse, F. Thiele, Time domain impedance modelling and applications, Procedia Engineering 6 (2010) $133-142$ 
[20] J. W. Meadows, A. K. Agrawal, Porous inserts for passive control of noise and thermo-acoustic instabilities in ldi combustion, Combustion Science and Technology 187 (2015) $1021-1035$.

[21] P. Tudisco, R. Ranjan, S. Menon, S. Jaensch, W. Polifke, Application of the time-domain impedance boundary condition to large-eddy simulation of combustion instability in a shear-coaxial high pressure combustor, Flow, Turbulence and Combustion 99 (2017) $185-207$.

[22] A. Rasheed, H. Hornung, A. Fedorov, N. Malmuth, Experiments on passive hypervelocity boundary-layer control using an ultrasonically absorptive surface, AIAA Journal 40 (2002) 481-489.

[23] A. Wagner, K. Hannemann, M. Kuhn, Ultrasonic absorption characteristics of porous carboncarbon ceramics with random microstructure for passive hypersonic boundary layer transition control, Experiments in Fluids 55 (2014) 1750.

[24] V. C. B. Sousa, D. Patel, J.-B. Chapelier, V. Wartemann, A. Wagner, C. Scalo, Numerical investigation of second-mode attenuation over carbon/carbon porous surfaces, Journal of Spacecraft and Rockets 56 (2019) 319 - 332.

[25] D. A. Kopriva, J. H. Kolias, A conservative staggered-grid chebyshev multidomain method for compressible flow, NASA ICASE Report 95-18, 1995.

[26] Y. Liu, M. Vinokur, Z. Wang, Spectral difference method for unstructured grids i: Basic formulation, Journal of Computational Physics 216 (2006) $780-801$.

[27] Z. J. Wang, Y. Liu, G. May, A. Jameson, Spectral difference method for unstructured grids ii: Extension to the euler equations, Journal of Scientific Computing 32 (2007) 45-71.

[28] G. May, A. Jameson, A spectral difference method for the euler and navier-stokes equations on unstructured meshes, AIAA Paper 2006-304, in: 44th AIAA Aerospace Sciences Meeting and Exhibit

[29] Y. Sun, Z. J. Wang, Y. Liu, High-order multidomain spectral difference method for the navier-stokes equations on unstructured hexahedral grids, Communications in Computational Physics 2 (2007) 310-333.

[30] K. V. Den Abeele, C. Lacor, Z. J. Wang, On the stability and accuracy of the spectral difference method., J. Sci. Comput. 37 (2008) 162 188.

[31] A. Jameson, A proof of the stability of the spectral difference method for all orders of accuracy, Journal of Scientific Computing 45 (2010) $348-358$.

[32] A. H. Mohammad, Z. J. Wang, C. Liang, Large eddy simulation of flow over a cylinder using high-order spectral difference method, Adv. Appl. Math. Mech 2 (2010) $451-466$.

[33] C. Liang, S. Premasuthan, A. Jameson, High-order accurate simulation of low-mach laminar flow past two side-by-side cylinders using spectral difference method, Computers \& Structures 87 (2009) 812 - 827.

[34] Y. Zhou, Z. Wang, Simulation of caa benchmark problems using high-order spectral difference method and perfectly matched layers, AIAA Paper 2010-838, in: 48th AIAA Aerospace Sciences Meeting Including the New Horizons Forum and Aerospace Exposition.

[35] J. Vanharen, G. Puigt, X. Vasseur, J.-F. Boussuge, P. Sagaut, Revisiting the spectral analysis for high-order spectral discontinuous methods, Journal of Computational Physics 337 (2017) 379 - 402.

[36] S. K. Lele, Compact finite difference schemes with spectral-like resolution, Journal of Computational Physics 103 (1992) 16 - 42.

[37] C. Bogey, C. Bailly, A family of low dispersive and low dissipative explicit schemes for flow and noise computations, Journal of Computational Physics 194 (2004) $194-214$.

[38] H. T. Huynh, A flux reconstruction approach to high-order schemes including discontinuous galerkin methods, AIAA Paper 2007-4079, in: 18th AIAA Computational Fluid Dynamics Conference.

[39] H. Gao, Z. Wang, A high-order lifting collocation penalty formulation for the navier-stokes equations on 2-d mixed grids, AIAA Paper 2009-3784, in: 19th AIAA Computational Fluid Dynamics.

[40] T. Haga, H. Gao, Z. J. Wang, A high-order unifying discontinuous formulation for the navier-stokes equations on $3 \mathrm{~d}$ mixed grids, Math. Model. Nat. Phenom. 6 (2011) 28-56.

[41] P. E. Vincent, P. Castonguay, A. Jameson, A new class of high-order energy stable flux reconstruction schemes, Journal of Scientific Computing 47 (2011) 50-72.

[42] V. Singh, S. Frankel, Wall-modeled implicit les of transitional flows using variable-order flux reconstruction method, AIAA Paper 2019-0645, in: AIAA Scitech Forum.

[43] T. Haga, S. Tsutsumi, S. Kawai, R. Takaki, Large-eddy simulation of a supersonic jet using high-order flux reconstruction scheme method, AIAA Paper 2015-0831, in: 53rd AIAA Aerospace Sciences Meeting.

[44] C. Liang, C. Cox, M. Plesniak, A comparison of computational efficiencies of spectral difference method and correction procedure via reconstruction, Journal of Computational Physics 239 (2013) 138 - 146.

[45] Z. J. Wang, H. T. Huynh, A review of flux reconstruction or correction procedure via reconstruction method for the navier-stokes equations, Mechanical Engineering Reviews 3 (2016) 15-00475-15-00475.

[46] J. W. Kim, D. Joo, Generalized characteristic boundary conditions for computational aeroacoustics, part 2, AIAA Journal 42 (2004) 47 - 55.

[47] C. S. Yoo, H. G. Im, Characteristic boundary conditions for simulations of compressible reacting flows with multi-dimensional, viscous and reaction effects, Combustion Theory and Modelling 11 (2007) 259-286.

[48] C. S. Yoo, Y. Wang, A. Trouvé, H. G. Im, Characteristic boundary conditions for direct simulations of turbulent counterflow flames, Combustion Theory and Modelling 9 (2005) $617-646$.

[49] V. Granet, O. Vermorel, T. Lonard, L. Gicquel, T. Poinsot, Comparison of nonreflecting outlet boundary conditions for compressible solvers on unstructured grids, AIAA Journal 48 (2010) $2348-2364$.

[50] A. Fosso P., H. Deniau, N. Lamarque, T. Poinsot, Comparison of outflow boundary conditions for subsonic aeroacoustic simulations, International Journal for Numerical Methods in Fluids 68 (2012) 1207 - 1233.

[51] Q. Liu, O. V. Vasilyev, Nonreflecting boundary conditions based on nonlinear multidimensional characteristics, International Journal for Numerical Methods in Fluids 62 (2010) $24-55$.

[52] N. Odier, M. Sanjos, L. Gicquel, T. Poinsot, S. Moreau, F. Duchaine, A characteristic inlet boundary condition for compressible, turbulent, multispecies turbomachinery flows, Computers \& Fluids 178 (2019) $41-55$.

[53] F. Monteghetti, D. Matignon, E. Piot, L. Pascal, Design of broadband time-domain impedance boundary conditions using the oscillatory- 
diffusive representation of acoustical models, The Journal of the Acoustical Society of America 140 (2016) 1663 - 1674.

[54] F. Monteghetti, D. Matignon, E. Piot, Energy analysis and discretization of nonlinear impedance boundary conditions for the time-domain linearized euler equations, Journal of Computational Physics 375 (2018) 393 - 426.

[55] E. J. Brambley, Well-posed boundary condition for acoustic liners in straight ducts with flow, AIAA Journal 49 (2011) 1272 - 1282.

[56] S. Rienstra, Impedance models in time domain, including the extended Helmholtz resonator model, in: 12th AIAA/CEAS Aeroacoustics Conference.

[57] M. Jones, W. Watson, T. Parrott, Benchmark data for evaluation of aeroacoustic propagation codes with grazing flow, AIAA Paper 2005-2853, in: 11th AIAA/CEAS Aeroacoustics Conference.

[58] Q. Douasbin, C. Scalo, L. Selle, T. Poinsot, Delayed-time domain impedance boundary conditions (d-tdibc), Journal of Computational Physics 371 (2018) $50-66$.

[59] C. Scalo, J. Bodart, S. K. Lele, Compressible turbulent channel flow with impedance boundary conditions, Physics of Fluids 27 (2015) 035107.

[60] K.-Y. Fung, H. Ju, Time-domain impedance boundary conditions for computational acoustics and aeroacoustics, International Journal of Computational Fluid Dynamics 18 (2004) $503-511$.

[61] G. P. A. Cassagne, J.-F. Boussuge, High-order method for a new generation of large eddy simulation solver, 2015.

[62] W. Hamri, Evaluation of the jaguar solver with benchmark test cases, 2015.

[63] D. H. Rudy, J. C. Strikwerda, A nonreflecting outflow boundary condition for subsonic navier-stokes calculations, Journal of Computational Physics 36 (1980) $55-70$.

[64] N. Lamarque, M. Porta, F. Nicoud, T. Poinsot, On the stability and dissipation of wall boundary conditions for compressible flows, International Journal for Numerical Methods in Fluids 62 (2010) 1134 - 1154

[65] C. Richter, F. H. Thiele, X. D. Li, M. Zhuang, Comparison of time-domain impedance boundary conditions for lined duct flows, AIAA Journal 45 (2007) $1333-1345$.

[66] S. Zhong, X. Zhang, X. Huang, A controllable canonical form implementation of time domain impedance boundary conditions for broadband aeroacoustic computation, Journal of Computational Physics 313 (2016) 713 - 725.

[67] M. O. Burak, M. Billson, L.-E. Eriksson, S. Baralon, Validation of a time- and frequency-domain grazing flow acoustic liner model, AIAA Journal 47 (2009) $1841-1848$. 